\title{
Aspects of Chiral Symmetry and the Lattice*
}

\author{
Michael Creutz \\ Physics Department \\ Brookhaven National Laboratory \\ Upton, NY 11973 \\ creutz@bnl.gov
}

\begin{abstract}
I explore the non-perturbative issues entwining lattice gauge theory, anomalies, and chiral symmetry. After briefly reviewing the importance of chiral symmetry in particle physics, I discuss how anomalies complicate lattice formulations. Considerable information can be deduced from effective chiral Lagrangians, helping interpret the expectations for lattice models and elucidating the role of the CP violating parameter $\Theta$. I then turn to a particularly elegant scheme for exploring this physics on the lattice. This uses an auxiliary extra space-time dimension, with the physical world being a four dimensional interface.
\end{abstract}

\section{Introduction}

Difficulties with chiral symmetry have plagued lattice gauge theory from the earliest days. Sometimes regarded as a technical problem associated with Fermion doublers, the issues delve deeply into quantum anomalies of field theory. My goal here is a pedagogical discussion of the interplay between chiral symmetry and the lattice. The discussion is biased towards my own, sometimes unconventional, point of view, and is not an attempt to cover the extensive and rapidly growing literature on the topic. Rather I hope to convince the reader of the deep consequences for the understanding of quantum field theory. I will be mainly qualitative, concentrating on non-perturbative issues. I will loosely intermingle considerations on the behavior of gauge theories as functions of Fermion masses with speculations on the obstacles to coupling gauge fields with chiral currents. While many profound topological issues arise in these connections, I will concentrate more on the physical interpretations.

The lattice plays a dual role in particle physics. On a practical level it is a tool for calculating non-perturbative phenomena. Here it has taught much about hadronic

\footnotetext{
* For Reviews of Modern Physics.
} 
physics, both in isolation and in terms of corrections to electro-weak phenomena. On a more conceptual level the lattice is a defining regulator for quantum field theory. The continuum theory is the zero lattice spacing limit of the lattice formulation. If this limit does not exist, can the continuum theory make sense? If unexpected phenomena occur as the limit is taken, these must be understood in the context of potential manifestations in the final theory. From this point of view, it is essential to understand all properties of the continuum theory in lattice language. Chiral symmetry has played a major role in the history of theoretical particle physics, but has a long and tortured history in lattice models. These difficulties with chiral issues on the lattice may well be revealing something deep.

I assume that the reader is familiar with the basics (Creutz, 1983) of lattice gauge theory, i.e. concepts such as gauge fields as elements of the gauge group on the links of a four dimensional lattice, a gauge action formed out of plaquette variables, and the exact local gauge invariance of the lattice theory. I also assume a basic familiarity with chiral symmetry in the continuum and the $S U(N) \times S U(N)$ symmetry of the strong interactions with $N$ massless quarks. I concentrate on the aspects that make formulating chiral symmetry on the lattice tricky, and will bypass several fascinating topics on the interplay of chiral symmetry breaking and confinement. These include anomaly matching conditions (Coleman and Grossman, 1982), the structure of low lying Fermionic states (Banks and Casher, 1980; Verbaarschot, 1994), and various models of the æether (van Baal, 1998). I will assume from the outset that confinement of quarks into mesons and baryons is a fundamental property of the strong interactions. I also will not discuss the rich interplay of chiral symmetry and finite temperature.

The next section provides an overview of the importance of chiral symmetry to particle physics. The following two sections discuss how quantum mechanical anomalies eliminate some symmetries from classical field theory, and show that these effects have analogues in simple band theory. Section V shows how ignoring these phenomena in lattice models can lead to multiplication of species. Here I mention some of the older traditional schemes for eliminating these difficulties. Section VI lists some of the generic schemes to restore the non-anomalous chiral symmetries through the use of large numbers of auxiliary fields.

To stress some of the physics that must arise in any correct formulation of chiral symmetry, several sections, VII through X, explore how anomalies introduce a possible $\mathrm{CP}$ violating parameter into the strong interactions. These sections emphasize how these 
effects are strongly dependent on the number of Fermion species. The physics of the anomaly results in a rather intriguing phase structure as a function of complex quark masses. Throughout this treatment effective chiral Lagrangians, another powerful nonperturbative tool, play a major role.

Returning to the lattice, section XI speculates on how these flavor dependent phenomenon may appear with standard Wilson lattice Fermions. Section XII shows that the picture of section XI is not quite complete, and explores the Aoki phase, a rather fascinating lattice artifact expected whenever a lattice action has explicit chiral breaking terms.

Section XIII reviews how anomalies are elegantly incorporated into effective Lagrangians through the use of an extra dimension. This is a partial motivation for the domain-wall Fermion approach introduced in Sections XIV through XVI. Section XVII gives a physical interpretation for anomalous processes in in this approach via a flow of Fermion states in the extra dimension. Sections XIII and XIX treat two contrasting schemes for incorporating the parity violation of the weak interactions into lattice gauge theory. Section XX derives an exact chiral identity for domain-wall Fermions, casting light on the distinction between singlet and non-singlet chiral currents.

Section XI briefly discusses recent efforts to eliminate the extra dimension and implement exact chiral symmetries directly on four dimensional systems. The final two sections contain speculations about the domain-wall Fermion idea and some concluding remarks. 


\section{Why chirality?}

If the lattice is the definition of a quantum field theory, why bother understanding chiral symmetry at anything but a superficial level? Just put the model on the computer, calculate the particle properties, and go home. This attitude, however, ignores a long history of fascinating developments tied to chirality in theoretical particle physics.

Lord Kelvin (1904) introduced the word "chiral," coming from the Greek word for hand, to refer to objects, such as hands, that are distinguishable under reflection. The most common use of the term is in chemistry, where a chiral molecule has a distinct mirror image. A carbon atom tetrahedrally bound to four different groups is the simplest example; tartaric acid with its two chiral centers is historically the most famous. In particle physics the term is adapted to distinguish massless particles by their helicity, i.e. their spin along the direction of motion.

For the particle physicist, chirality is deeply entwined with the Lorentz group. Indeed, massless representations are qualitatively different from their massive counterparts. Without mass, the helicity of a particle is invariant under boosts and rotations. The helicity becomes a Lorentz invariant concept. When a spin $1 / 2$ Fermion is coupled minimally to a gauge theory, this helicity conservation survives interactions, and currents associated with left and right handed particles are naively separately conserved. The Fermion fields naturally break into two independent parts, $\psi_{R} \equiv \frac{1}{2}\left(1+\gamma_{5}\right) \psi$ and $\psi_{L} \equiv \frac{1}{2}\left(1-\gamma_{5}\right) \psi$. With only one spatial dimension the concept is even simpler. A massless excitation traveling at the speed of light can never be overtaken. Thus a particle moving to the right does so in all frames, and the fields break up into left and right moving parts.

Infamous anomalies complicate this simple picture. Indeed, with gauge interactions present, not all axial currents can be conserved. This lies at the root of much fascinating physics, entwined with such issues as strong $C P$ violation, the presence of an unanticipated parameter $\Theta$, the so called $U(1)$ problem, the mass of the $\eta$ meson, etc. The lattice, by removing all infinities from the problem, forces the study of these effects at a deep and fundamental level.

There are at least three easily identifiable reasons for particle physicists to study chiral symmetry. First, the observed world is chiral. Only left handed neutrinos emerge from beta decays. The underlying theory must incorporate parity violation. In the standard model this appears through chiral couplings of the gauge fields to Fermions. In the massless limit, the intermediate vector Bosons of the weak interactions couple only to left handed 
helicities. It is essential that any fundamental formulation incorporate this asymmetric coupling. Perturbatively this has not proven to be a major problem, at least if anomalies are appropriately canceled. However for the lattice, a direct non-perturbative scheme for defining field theories, this anomaly cancellation enters at a rather deep level. Indeed, there still is not a universal agreement on how to formulate the full standard model on the lattice. Minefields of unproven technical details lie scattered along the paths of all existing approaches.

A lattice formulation of the standard model is perhaps a rather technical issue, not particularly relevant for observable phenomena. The weak interactions are weak, and perturbation theory works extremely well for any experimental observations. The main exception lies in attempts to understand the early universe, when the temperature was of the order of the weak scale. At this time non-perturbative phenomena may have played a major role in the generation of the baryon number asymmetry in the universe.

The second reason to explore chiral symmetry in a lattice context is more phenomenological, and is increasingly showing its import to ongoing experimental results. This has its roots in the strong interactions and the low mass of the pion relative to other hadrons. The underlying classical Lagrangian for the quark confining theory of the strong interactions has extra symmetry in the massless limit. All terms in this limiting Lagrangian independently conserve the number of left handed and right handed Fermions. With massless quarks this is an exact classical symmetry of the theory. There exists a vast repository of literature on how this symmetry is spontaneously broken by the vacuum, with the pions being approximate Goldstone Bosons. The pion mass is small because the two lightest quarks have small masses. Indeed, the phenomenology of chiral symmetry-breaking works remarkably well even when the strange quark is also treated as light.

The immense success of the phenomenology based on spontaneous chiral symmetry breaking compels an effort to understand its role on the lattice. To ignore the chiral issues, simply calculating hadronic phenomenology as functions of the quark masses and adjusting to fit experiment, deprives one of much understanding of how the particles derive their masses and the vast successes of the effective chiral Lagrangian approach to low energy meson interactions.

Finally, chiral symmetry plays a crucial role in most proposed extensions of the standard model. These often involve unifications at scales much larger than those of ordinary hadronic physics. In the process of renormalizing and projecting out the low energy the- 
ory, it appears peculiar that the physical particles in the laboratory are so much lighter than these possibly more fundamental scales. To argue around this unnaturalness, chiral symmetry serves as a protector of light masses, which only receive multiplicative renormalizations. A massless particle should remain so under renormalization. This is also one of the main motivations for super-symmetry; by relating fundamental scalars such as the Higgs meson to Fermionic partners, chiral symmetry can restrict the renormalization of their masses as well. 


\section{Anomalies}

It is well known that not all classical symmetries survive quantization. The most basic example, the scale anomaly, has been so fully absorbed into the lattice lore that it is rarely mentioned. When the quark masses vanish, the classical Lagrangian for the strong interactions contains no dimensional parameters. But the quantum theory is supposed to describe baryons and mesons, and the lightest baryon, the proton, definitely has mass. This is understood through the phenomenon of "dimensional transmutation," wherein the classical coupling constant of the theory is traded, through the process of renormalization, for an overall scale parameter (Coleman and Weinberg, 1973).

This scale anomaly is central to understanding asymptotically free gauge theories; so, let me briefly repeat the basic words. On quantization of the initial theory, the usual divergences of loop diagrams are encountered. These are removed by an ultraviolet cutoff, but the latter inherently involves a length scale. To remove the cutoff one must adjust, or "renormalize" the bare parameters of the theory so that physical parameters remain finite. For the strong interactions with massless quarks, the only bare parameter is the gauge coupling $g$. This quantity becomes a function of the cutoff $\Lambda$. The coupling "runs," following the behavior dictated by the "renormalization group equation." Because of asymptotic freedom, the bare coupling runs to zero as the cutoff is removed.

In this way of thinking, any physical mass parameter, say the proton mass, takes the form of a coupling dependent factor times the only dimensional parameter around, the cutoff

$$
m_{p}=f_{p}(g) \Lambda .
$$

The factor $f_{p}$ goes to zero as the cutoff $\Lambda$ goes to infinity, leaving the product finite. Given two different physical masses, say $m_{\rho}$ and $m_{p}$ for the rho and the nucleon, the scale drops out of their ratio. Thus

$$
\frac{m_{\rho}}{m_{p}}=\frac{f_{\rho}(g)}{f_{p}(g)}
$$

is a function only of the bare coupling. The physical value is this ratio evaluated at zero bare coupling. If the quantum field theory is well defined, this final result must be independent of the scheme used for regularization. At this point there are no more parameters to play with. Any dimensionless ratio is completely determined, with no free parameters. If a calculation gets some ratio of masses wrong, then either the calculation or the theory is incorrect. The net outcome is really quite amazing — a fully parameter free 
quantum field theory. It is no longer scale invariant, but has an overall scale fixed by the physical particle masses. The ratios of any of these masses should be exactly determined, and calculable via lattice simulations.

This discussion applies to the massless theory. When the quark masses are turned on, these, measured in units of the above generated scale, provide additional parameters to adjust. What determines their values is one of the outstanding questions of particle physics. Conceivably they also come from some sort of "dimensional transmutation" in a higher level theory, but this goes well beyond the context of this review.

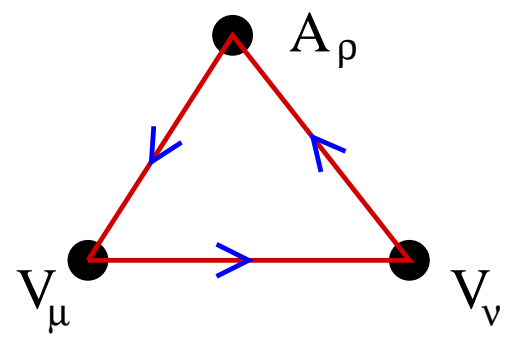

Fig. 1. In four dimensions, regularization of triangle diagrams involving both axial and vector currents allows only one of the two to be conserved, even as the Fermion mass goes to zero.

The scale anomaly is not the only symmetry of the strong interactions of massless quarks that is lost upon quantization. The most famous, and central to this review, are the anomalies in the axial vector Fermion currents (Bell and Jackiw, 1969; Adler, 1969). Working in a helicity basis, the classical Lagrangian has no terms to change the number of left or right handed Fermions. On quantization, however, these numbers can not be separately conserved. Technically this comes about because of the famous triangle diagram of Fig. (1) . This introduces a divergence to the theory which requires regularization via a dimensional cutoff. For the strong interactions this cutoff is removed so that the vector current, representing total Fermion number, is conserved. But if this choice is taken, then the axial current, representing the difference of right and left handed Fermion numbers, cannot be. There is a freedom in choosing which currents are conserved; however, in a gauge theory, consistency requires that gauge fields couple only to conserved currents.

In the standard model, anomalies require some time honored conservation laws to be violated. The most famous example ('t Hooft, 1976a,b) is baryon number, which in the standard model is sacrificed so that the chiral currents that couple to the vector Bosons are conserved. Baryon violating semi-classical processes have been identified and must be 
present, although at a very low rate. While not of observable strength, at a conceptual level any scheme for non-perturbatively regulating the standard model must either contain baryon violating terms (Eichten and Preskill, 1986) or extend the model to cancel these anomalies with, say, mirror species. Furthermore, this consistency has non-trivial implications for the allowed species of Fermions. To conserve all the gauged currents of the standard model requires the cancellation of all potential anomalies in currents coupled to gauge fields. In particular, the standard model is not consistent if either the leptons or the quarks are left out. This connection between quarks and leptons is a deep subtlety of the theory and must play a key role in placing the theory on a lattice.

For the strong interactions, the chiral anomaly has dramatic consequences for the low lying hadronic spectrum. As mentioned earlier, the light nature of the quark masses gives rise to the interpretation of pions as approximate Goldstone Bosons associated with a spontaneous breaking of chiral symmetry. But mesons associated with broken chiral currents need not be as light. Considering only the up and down quarks as light, the eta meson is heavier than the pion even though it is constructed from the same quarks. Under the eightfold way involving the strange quark, it is the $\eta^{\prime}$ meson which is anomalously heavy. 


\section{Band theory and anomalies}

The necessity for chiral anomalies is easily understood in lower dimensions. In a world with only one time and one space dimension, there is no spin, and the concept of chirality reduces to a distinction between particles moving in one direction relative to the other. If a particle is massless, the inability to boost by more than the speed of light indicates that a right moving particle is so in all Lorentz frames. For free particles the number of left movers and the number of right movers are separately conserved quantities.

A gauge interaction would at first sight seem to preserve this symmetry since the classical form of the current $\bar{\psi} \gamma_{\mu} \psi=\bar{\psi}_{R} \gamma_{\mu} \psi_{R}+\bar{\psi}_{L} \gamma_{\mu} \psi_{L}$ separates into two independent terms. However, in analogy with the triangle diagram in four dimensions, there is a bubble diagram which cannot be regulated so that both the vector and axial currents are conserved.
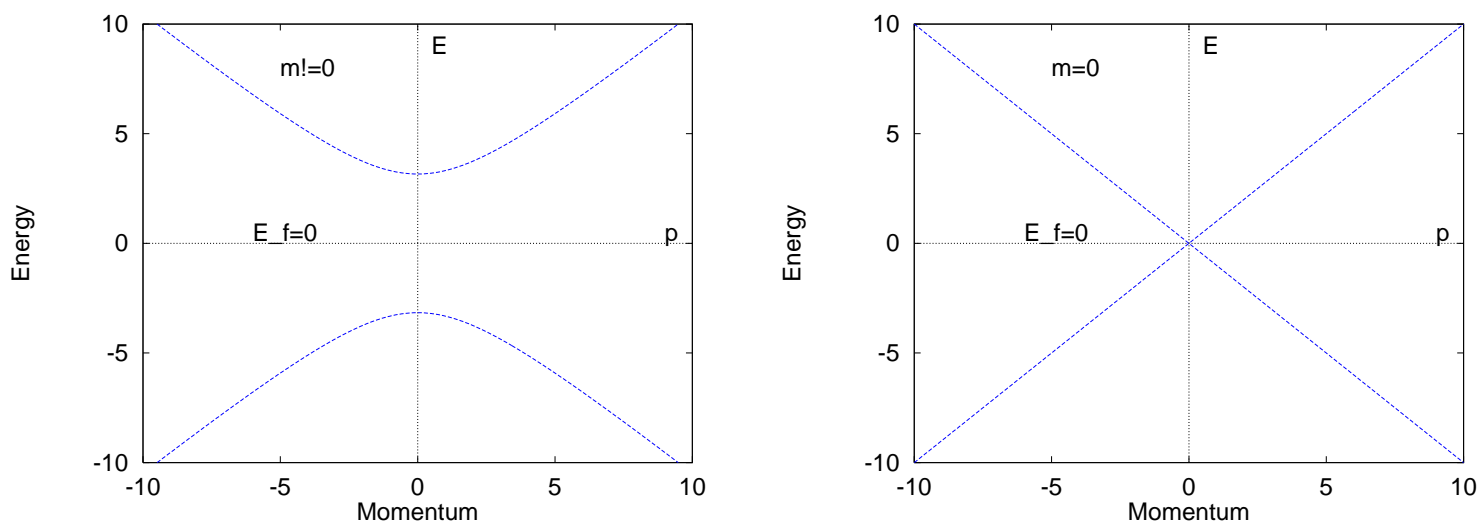

Fig. 2. A massive Fermion corresponds to an insulating vacuum. A massless Fermion gives a conductor. The induction of currents in this conductor corresponds to the chiral anomaly.

In a Hamiltonian language, the anomaly arises from a sliding of states in and out of the Dirac sea (Ambjorn, Greensite, and Peterson 1983; Holstein, 1993). The mechanism can be seen intuitively from simple band theory. Consider a massive Fermion with energy spectrum $E=\sqrt{p^{2}+m^{2}}$, as sketched in Fig. (2) . In the Dirac picture, there is a filled sea of negative energy states, in which holes represent anti-particles. The Fermi level of the vacuum lies at $E=0$, between these two bands, and this is the classic picture of an insulator.

Now for the massless case the energy spectrum reduces to $E= \pm p$. The right moving particles are represented by the increasing branch, $E=p$ and the left movers occupy the 
decreasing branch. I note in passing that an anti-particle of a right-mover also moves to the right. It corresponds to a hole in the negative energy, negative momentum spectrum. Thus it has an effective positive momentum. This is in contrast to the three dimensional case where an anti-particle has opposite helicity.

In the band theory language, the theory no longer has a gap. It is a conductor. The current is proportional to the number of right movers minus the number of left movers. Here is where the anomaly comes in: electric fields can induce currents in a conductor. Thus an electric field will change the relative number of right and left movers. Without the anomaly, wires would not conduct and transformers would not work.

The mechanism of the anomaly is particularly clear in this Dirac sea picture. An electric field pushes on the filled levels, increasing their momentum. As the momentum changes, a negative energy state can acquire positive energy. The anomaly is not due to a left moving state jumping to a right mover, but rather a shifting of the negative energy states up or down depending on the branch.

This picture can be generalized to three dimensions. First the Fermions are subjected to a magnetic field that confines them transversely into Landau levels. The lowest of these Landau levels has precisely the spectrum of a one dimensional model, where that dimension is the distance along the magnetic field. Then an applied electric field parallel to that direction can induce currents in that level. The anomaly is proportional to $\vec{E} \cdot \vec{B} \sim \tilde{F}_{\mu \nu} F_{\mu \nu}$.

Note that this picture of level flow relies on an infinite number of states on each branch. A new right mover arises from a shift out of an infinite negative energy sea, while an anti left-mover is a new hole arising from a depression of the Dirac sea. This discussion of how the anomaly works also hints at why it is difficult in a lattice approach. With such a regulator, the momentum is bounded by the cutoff and the spectral branches cannot go to infinity; the maximum allowed momentum on a lattice of spacing $a$ is $\pi / a$. When the states slide up under the influence of a field, a mechanism must be provided to absorb one state from the top. Generally this requires either an explicit breaking of the chiral symmetry or another infinity. 


\section{Lattice Fermions}

That there is a difficulty with lattice Fermions was realized quite early. Take the simple continuum Lagrangian for a free massless Dirac field

$$
S_{\mathrm{cont}}=\int d^{4} x \bar{\psi} i \not \partial \psi
$$

and use a simple difference scheme for the derivative on an $L^{4}$ lattice

$$
S_{\text {lattice }}=\frac{1}{2} \sum_{j} \sum_{\mu=0}^{4} i \bar{\psi}_{j} \gamma_{\mu}\left(\psi_{j+e_{\mu}}-\psi_{j-e_{\mu}}\right)
$$

where $e_{\mu}$ represents a unit step in the $\mu$ direction. To remove explicit factors of the lattice spacing, I have rescaled the Fermionic fields by a factor of $\sqrt{a^{3}}$; thus, one would relate the continuum field and the lattice one by $\psi_{j} \leftrightarrow \sqrt{a^{3}} \psi(x=j a)$. Going to momentum space on a periodic lattice,

$$
\begin{aligned}
\psi_{j} & =\frac{1}{L^{2}} \sum_{q} e^{-2 \pi i j \cdot q / L} \tilde{\psi}_{q} \\
\tilde{\psi}_{q} & =\frac{1}{L^{2}} \sum_{j} e^{2 \pi i j \cdot q / L} \psi_{j}
\end{aligned}
$$

the lattice action becomes diagonal

$$
S_{\text {lattice }}=\sum_{q} i \overline{\tilde{\psi}}_{q} \gamma_{\mu} \sin \left(2 \pi q_{\mu} / L\right) \tilde{\psi}_{q}
$$

and I can explicitly evaluate the propagator

$$
\left\langle\bar{\psi}_{j} \psi_{k}\right\rangle=\sum_{q} e^{-2 \pi i q \cdot(j-k) / L} D(q)
$$

where

$$
D(q)=\frac{1}{i \sum_{\mu} \gamma_{\mu} \sin \left(2 \pi q_{\mu} / L\right)} .
$$

For small momentum, this reduces to the conventional propagator $\frac{1}{i \not p}$ with the identification $p=2 \pi q / L a$ and the continuum position $x=a j$.

Because of the properties of the sine function, the free quark propagator in Eq. (8) has poles not only at $p_{\mu}=0$ but also when any component of the momentum is of magnitude $\pi / a$. Since the natural range of momentum can be taken as $0 \leq p_{\mu}<2 \pi / a$, in four dimensions there are sixteen poles representing 16 Fermionic species. 
These degenerate species have different chiralities (Karsten and Smit, 1981). Since the slope of the sine function is negative at $\pi$, when a component of momentum is near that value, the Fermion effectively uses a gamma matrix that differs by a sign. For the matrix $\gamma_{5}=\gamma_{0} \gamma_{1} \gamma_{2} \gamma_{3}$, half the species use the opposite sign. The naive axial current, $\bar{\psi} \gamma_{\mu} \gamma_{5} \psi$ represents a flavor non-singlet current in the resulting 16 flavor theory. Such currents are not required to be anomalous, and this theory has good chiral properties, albeit with more flavors than naively expected.

A similar doubling problem should be expected in any fully regulated local formulation that ignores anomalies but attempts to keep the classical chiral symmetries. Something must go wrong in the path integral, and in this case it is the appearance of multiple species in the quantum theory. Doubling is more than simply an artifact of the lattice action. This phenomenon has a topological interpretation as emphasized in early papers on the topic (Nielsen and Ninomiya, 1981a,b,c), and recently generalized in (Shamir, 1993a).

I note in passing that a single generation in the standard model has eight Fermionic species: three colors of quark doublet and one lepton doublet (allowing for a sterile righthanded neutrino). It is tempting to relate this power of two with some sort of doubling, and indeed this full structure of quarks and leptons is involved in the electroweak anomaly cancellation. While this view of Fermion multiplicities within a Fermion generation is unconventional, it is sufficiently amusing that I will explore it a bit more in a later section.

With the goal of eliminating doubling as a lattice artifact, much effort has gone into more complex Fermionic actions. One popular approach (Kogut and Susskind, 1975) staggers the four components of the Fermions over separate lattice sites. This removes one factor of four from the doubling, leaving four species. The resulting theory has one remaining exact chiral symmetry. The approach has been extremely popular for simulations because 1.) the Fermion matrices are smaller, and 2.) the remnant chiral symmetry protects the Fermion mass from additive renormalization. The main penalty of this approach is that flavor symmetry is sacrificed; with four flavors there is only one exact Goldstone Boson in the chiral limit and the other pions remain massive. In later sections I will argue for a strong physical dependence on the number of light quark flavors. Much of this physics may be hidden by this sacrifice of flavor symmetry.

The other traditional way (Wilson, 1977) to deal with this "problem" destroys the chiral symmetry at the outset. The doublers occur at non-zero momentum, and thus can be given a mass via a term that can be thought of as a momentum dependent mass. Having 
this vanish at zero momentum keeps the desired Fermion light, while allowing it to be large at the doubling points with $p_{\mu}=\pi / a$ removes the doublers. This can be accomplished with only nearest neighbor hoppings via the free lattice action

$$
S=\sum_{q, \mu} 2 K \overline{\tilde{\psi}}\left(i \gamma_{\mu} \sin \left(2 \pi q_{\mu} / L\right)+r\left(1-\cos \left(2 \pi q_{\mu} / L\right)\right) \tilde{\psi}+m \tilde{\tilde{\psi}} \tilde{\psi}\right.
$$

Here I have explicitly added a mass term and made the nearest neighbor coupling proportional to a "hopping parameter" $K$. In position space this reads

$$
S=K \sum_{j, \mu} \bar{\psi}_{j}\left(r+\gamma_{\mu}\right) \psi_{j+e_{\mu}}+\bar{\psi}_{j}\left(r-\gamma_{\mu}\right) \psi_{j-e_{\mu}}+M \sum_{j} \bar{\psi}_{j} \psi_{j}
$$

where the diagonal terms have been combined to give $M=8 K r+m$. This "Wilson Fermion" action indeed solves the doubling problem, leaving only one light Fermion species. Note that when the "Wilson parameter" $r$ is unity the hopping terms involve projection operators $1 \pm \gamma_{\mu}$. This simplifies many algebraic manipulations and is the most popular choice. The two parameters $M$ and $K$ are the natural ones in lattice language, and relate to the physical mass and lattice spacing.

In this scheme the price paid for removing the doublers is any exact chiral symmetry in the cutoff theory. One consequence is that when interactions are turned on, renormalizations may be required which involve operators that explicitly break chiral symmetry. In particular, the bare mass can receive an additive renormalization. There is nothing to protect a massless Fermion, so preserving a light pion mass will require finely tuning a mass counter-term. For the strong interactions there is nothing in principle known to be wrong with such an approach, and indeed one philosophy is to just do this renormalization, fitting particle masses and seeing that the final spectrum comes out acceptably. If one does not care about chiral issues, the Wilson approach is a perfectly acceptable regularization for Fermionic fields.

For a chiral gauge theory, such as the standard model, this approach is less clear. The breaking of chiral symmetry inherent in the Wilson term will break the gauge symmetry, introducing an infinite number of possible gauge variant counter-terms. This is a rather unpleasant situation which would be nice to avoid. Whether that is possible is still unresolved, although extensive studies using gauge fixing in conjunction with the lattice are ongoing (Borelli, Maiani, Sisto, Rossi, and Testa, 1990; Alonso, Boucaud, Corés, and Rivas, 1991; Bock, Golterman, and Shamir, 1998a,b). 


\section{An infinity of Fermions}

The last few years have seen an intense revitalisation of interest in chiral symmetry in the lattice context. Much of this was sparked by Kaplan (Kaplan, 1992) but the ideas have spread and joined with several other schemes. Indeed, (Narayanan and Neuberger, 1993a,b, 1994, 1995; Randjbar-Daemi and Strathdee, 1996a,b) emphasized the common feature that for the anomaly to work in a lattice theory, there must be some mechanism to absorb the Fermionic modes involved in anomalous processes. In the band theory language discussed earlier, for a state upwardly moving in energy not to be accompanied by a lowering one of the same chirality, it must be absorbed somewhere. In the continuum it comes and goes from the infinite Dirac sea. On a lattice with a finite number of degrees of freedom this is impossible. This suggests extending the theory with an infinite reservoir of Fermionic states. Indeed, one point of view is that any chiral gauge formulation on the lattice that preserves all the chiral symmetries must have an infinite number of auxiliary states in the Fermionic sector. In this sense all the recent schemes do something like this, differing only in where these states appear in the formalism.

Kaplan's suggestion has become known as the domain-wall approach. This involves allowing the Fermions to move in an extra space-time dimension. The low energy states are surface modes bound to a four dimensional surface of the underlying five dimensional system. As energies increase, the states penetrate further into the extra dimension, which absorbs the upwardly rising states of anomalous processes. But as far as low energies are concerned, the system appears four dimensional. I will discuss this mechanism in considerably more detail in later sections, but for now I note that an important part of the construction is that the gauge fields themselves do not observe this new coordinate, it is only for Fermions. In some sense the extra dimension is not really physical, and might be thought of as an "internal symmetry" or "flavor" space. As the size of this extra space goes to infinity, the consequences of chiral symmetry should become exact.

Frolov and Slavnov (1994) present a more abstract description building directly on an infinite tower of auxiliary states. In an extensive sequence of papers Neuberger and Narayanan (1993a,b, 1994, 1995) unified this approach with that of Kaplan, emphasizing how the effect of the bottom of the tower can be compactly represented as an overlap of two states in an auxiliary Hilbert space, effectively the space of a transfer matrix in the extra dimension.

Another scheme, a bit less explored, involves a finer lattice for the Fermions and a 
double limit, with the Fermion lattice spacing going to zero first (Hernandez and Sundrum, 1995; Hsu, 195; Bodwin, 1995; Hernandez and Boucaud, 1998). A variation on this treats the Fermions directly as continuum fields (Gockeler, Kronfeld, Schierholz and Wiese, 1993; Kronfeld, 1995; 't Hooft, 1995), acting under the influence of the gauge fields interpolated from the courser gauge lattice. A closely related procedure involves taking continuum Fermion fields and expanding in a complete set of basis functions motivated by a lattice holding the gauge fields (Friedberg, Lee, and Pang, 1994). On truncation the doubling returns, but as this basis goes to infinity one again has an infinite tower of Fermionic states. These approaches are a sharpening of a much older scheme based on directly using the continuum Fermion propagator (Drell, Weinstein, and Yankielowicz, 1976; Svetitsky, Drell, Quinn, and Weinstein, 1980). Whether these approaches are practical remains controversial; however they do share the property of introducing a large number of additional degrees of freedom over naive Fermion approaches.

My later presentation will concentrate on the domain wall approach. Nevertheless, I become more general in one section where I briefly discuss a promising class of schemes based on the Ginsparg-Wilson (1982) relation to generalize the continuum symmetries to an exact lattice symmetry. This approach parallels continuum discussions in diverting the complications of anomalies appear to the Fermionic measure. 


\section{Strong CP violation}

As discussed above, scale anomalies reduce the number of physically independent dimensionless parameters in the quark confining dynamics of quarks and gluons. For massless quarks, dimensional transmutation leaves no undetermined continuous parameters; the theory only depends the gauge group and the number of Fermion flavors.

With masses for the quarks, the number of parameters increases. Naively there is one additional mass parameter for each quark. However, due to chiral anomalies, one more parameter, usually called $\Theta$, is hidden in the phases in these quark masses. If non-zero, this parameter gives rise to CP violating processes in the strong interactions. Such appear to be extremely small in nature, suggesting that this parameter may vanish. In a grand unified context this raises puzzles since CP violation is present in the weak interactions and has no particular reason to be small. Possible resolutions to this problem go beyond the scope of this paper; for reviews see Turner (1990) and Raffelt (1990).

For the lattice it is crucial that any Fermion formulation be able to account for this parameter. To define a phase for a single Fermion mass, break the naive mass term into two parts

$$
\bar{\psi} \psi=\bar{\psi}_{L} \psi_{R}+\bar{\psi}_{R} \psi_{L}
$$

where the left and right parts are eigenstates of $\gamma_{5}$

$$
\psi_{R, L}=\frac{1}{2}\left(1 \pm \gamma_{5}\right) \psi_{R, L}
$$

Then a generic complex mass term has the form

$$
\bar{\psi}_{L} M \psi_{R}+\bar{\psi}_{R} M^{*} \psi_{L}
$$

with $M$ a complex number, i.e. of form $M=m e^{i \theta}$. Another convenient form for the mass term is

$$
m_{1} \bar{\psi} \psi+i m_{2} \bar{\psi} \gamma_{5} \psi
$$

so that $M=m_{1} \cos (\theta)+i m_{2} \sin (\theta)$.

At the classical level the phase of the quark mass is easily removed by a chiral rotation. The change of variables

$$
\begin{aligned}
& \psi \rightarrow e^{i \theta \gamma_{5} / 2} \psi \\
& \bar{\psi} \rightarrow \bar{\psi} e^{i \theta \gamma_{5} / 2}
\end{aligned}
$$


takes

$$
M \rightarrow e^{i \theta} M
$$

Since this is just a redefinition, if the path integral measure is invariant under the same phase change, theta can be rotated away. However, here the chiral anomalies come into play. Flavor non-singlet axial symmetries are believed to be preserved; so, one can eliminate any non-singlet phases in the mass. However one overall phase always remains. Using the anomaly, this can be moved from the quark masses into pure gauge terms of the action; indeed, the latter form is the starting point for most conventional discussions of $\Theta$ in terms of topological structures in the gauge sector ('t Hooft, 1986).

How this physics manifests itself in a theoretical formalism depends on approach. With a Pauli-Villars regulator, the heavy auxiliary field has a phase in its mass; the theta parameter is the relative phase between the regulator mass and that of the physical Fermion. In other continuum schemes the phase is often pushed into the path integral measure (Fujikawa, 1979).

In usual lattice approaches, the Fermion measure is a direct product of discrete Grassmann integrals, leaving no room in the measure for forbidding the change of variables alluded to above. However with Wilson Fermions we have the added Wilson term, which is itself of a mass like form. Theta then becomes a relative phase between the Wilson term and the explicit mass (Seiler and Stamatescu, 1982).

For another angle on the meaning of theta, it is useful to think in terms of effective chiral Lagrangians ('t Hooft, 1986; Creutz, 1995a; Evans, Hsu, Nyffeler, and Schwetz, 1997; Smilga, 1999; Tytgat, 1999). This temporarily leads away from the lattice, but gives insight into the expected structure of Wilson Fermions with complex mass. Also, the chiral Lagrangian approach will be useful in later sections for understanding other phenomena of direct relevance to the lattice, such as the Aoki phase and the Wess-Zumino motivation for exploring higher dimensions.

The phase diagram in the $\left(m_{1}, m_{2}\right)$ plane strongly depends on the number of Fermion flavors. In later sections I will be more explicit, but let me briefly summarize the qualitative picture. With a single species, a first-order phase transition line runs down the negative $m_{1}$ axis, starting at non-zero $m_{1}$. This is sketched in Fig. (3) . For two flavors there are two first-order phase transition lines, starting near the origin and running up and down the $m_{2}$ axis. For degenerate quarks these transitions meet at the chiral limit of vanishing Fermion mass; a small flavor breaking can separate the endpoints of these first-order lines. 


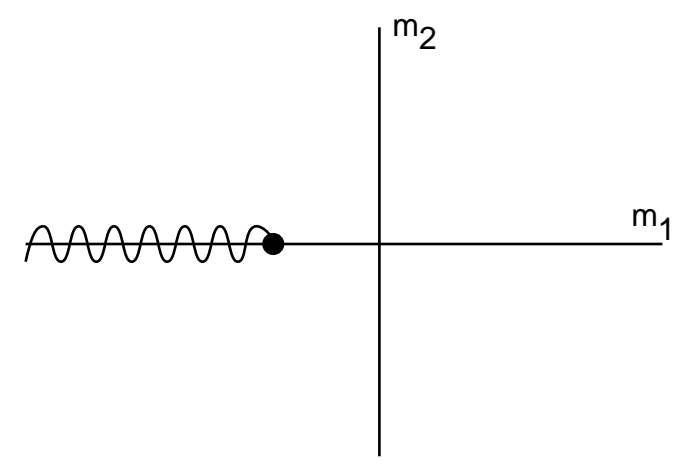

Fig. 3. The phase diagram for the one flavor case. The wavy line represents a first-order phase transition, along which $i \bar{\psi} \gamma_{5} \psi$ acquires an expectation value. The end point of this transition line is renormalized away from the origin towards negative $m_{1}$.

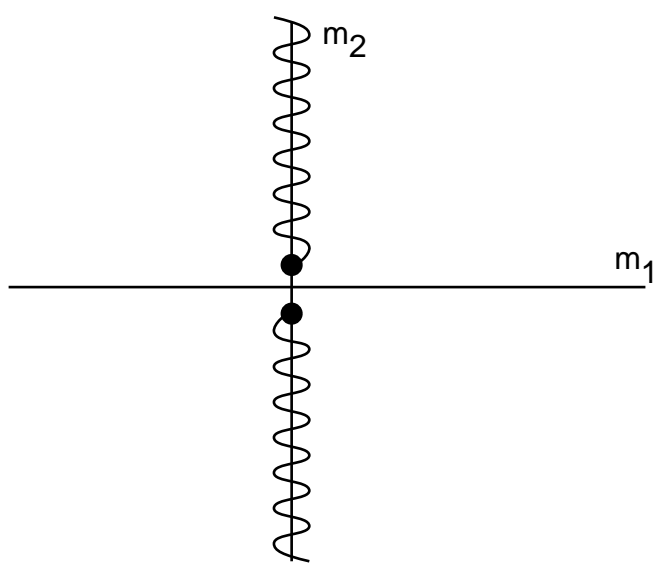

Fig. 4. The two flavor phase diagram. First-order lines run up and down the $m_{2}$ axis. The second order endpoints of these lines can be separated by flavor breaking terms. The chiral limit is pinched between these endpoints.

This is sketched in Fig. (4). With $N_{f}>2$ flavors, the $\left(m_{1}, m_{2}\right)$ plane has $N_{f}$ first-order phase transition lines pointing at the origin. The conventionally normalized parameter $\Theta$ is $N_{f}$ times the angle to a point in this plane, and these transition lines are each equivalent to $\Theta$ being $\pi$.

Whenever the number of flavors is odd, a first-order transition runs down the negative $m_{1}$ axis. Along this line there is a spontaneous breaking of $\mathrm{CP}$, with the natural order parameter being $\left\langle i \bar{\psi} \gamma_{5} \psi\right\rangle$. This possibility of a spontaneous breakdown of parity was noted some time ago by Dashen (1971) and has reappeared at various times in the lattice context 
(Smit, 1980; Aoki, 1989; Aoki and Gocksch, 1992). Any valid algorithm for dealing with an odd number of flavors must be able to distinguish the sign of the mass. The following three sections give detailed continuum arguments for this general structure, dealing successively with the three, one, and two flavor cases. 


\section{Three flavors}

To see how the above picture arises naturally in a chiral Lagrangian approach, start with the three flavor case. I begin with a lightning summary of effective theories of Goldstone Bosons. Suppressing other indices, consider left and right handed quark fields $\psi_{L}^{a}, \psi_{R}^{a}$ with a flavor index a running from one to three. Spontaneous chiral symmetry breaking appears in a non-vanishing expectation

$$
\left\langle\bar{\psi}_{L}^{a} \psi_{R}^{b}\right\rangle=v g^{a b}
$$

The vacuum is not unique, and is labeled by $g^{a b}$, an element of $S U(3)$. The basic $S U(3)_{L} \otimes$ $S U(3)_{R}$ chiral symmetry is realized via the global transformation

$$
g \rightarrow g_{L}^{\dagger} g g_{R}
$$

Picking $g=I$ for a standard vacuum, the 8 fields $\pi^{\alpha}$ which excite the Goldstone modes are nicely parametrized by

$$
g=e^{i \lambda^{\alpha} \pi^{\alpha} / F_{\pi}}
$$

where the matrices $\lambda^{\alpha}$ are a set of generators for the group $S U(3)$ and are normalized $\operatorname{Tr} \lambda^{\alpha} \lambda^{\beta}=2 \delta^{\alpha \beta}$

Effective Lagrangians start from the assumption that in the low energy limit only the Goldstone excitations are important; one considers an effective theory depending on $g$ alone. For low momenta, this theory is expanded in terms of increasing numbers of derivatives. To lowest order the effective Lagrangian is

$$
L=\frac{F_{\pi}^{2}}{4} \operatorname{Tr}\left(\partial_{\mu} g^{\dagger} \partial_{\mu} g\right)
$$

This is invariant under the global symmetry of Eq. (18). The parameter $F_{\pi}$ is related to the pion decay, which occurs through the axial vector coupling to the intermediate weak Boson. The phenomenological value is $F_{\pi}=93 \mathrm{MeV}$.

To give the quarks masses, add an explicit symmetry-breaking term. For degenerate quarks the simplest possibility adds to $L$ a potential term

$$
V=-\frac{m}{v} \operatorname{Re} \operatorname{Tr} g
$$

Minimizing $V$ for positive $m$ selects the standard $g=I$ to represent the vacuum. The pions are no longer massless, but acquire a mass proportional to the square root of the 
quark mass. For $S U(2),-I$ is an element of the group, so changing the sign of the mass simply induces a rotation to a new vacuum represented by $g=-I$. However $-I$ is not an element of $S U(3)$, requiring a somewhat more detailed analysis.

This leads me to a digression on some details of the $S U(N)$ group manifold. In particular, where is $\operatorname{Re} \operatorname{Tr}(g)$ extremal? At such a point, first order changes in $g$ must vanish; in particular

$$
0=\left.\frac{d}{d \epsilon^{\alpha}} \operatorname{Re} \operatorname{Tr}\left(g e^{i \lambda^{\beta} \epsilon^{\beta}}\right)\right|_{\epsilon=0}=i \operatorname{Tr}\left(g-g^{\dagger}\right) \lambda^{\alpha} .
$$

The $\lambda$ matrices are almost a complete set, only the identity is missing. This indicates that $g-g^{\dagger}$ is proportional to the identity; thus I write

$$
g-g^{\dagger}=i s I
$$

where the constant $s$ is yet to be determined.

Since $\operatorname{Tr} g$ is a class function, the extrema being discussed are by definition classes in the group. Given some extremal point $g$, all elements of form $h^{\dagger} g h$, with $h$ an arbitrary group element, are in the same equivalence class. Using this freedom, define a standard member of the class by diagonalizing $g$ and ordering the diagonal elements by increasing magnitude of the real part. After doing this, the diagonal elements are phases, and by Eq. (23) all have the same imaginary part. The diagonals must all be taken from the two numbers $c+i s$ or $-c+i s$ with $c=\sqrt{1-s^{2}}$.

In general there are a variety of such extremal elements, some of which are maxima, some minima, and some saddle points of ReTrg. To distinguish them, look at the second variation

$$
\left.\frac{d^{2}}{d \epsilon^{2}} \operatorname{Re} \operatorname{Tr}\left(g e^{i \lambda \epsilon}\right)\right|_{\epsilon=0}=-\operatorname{Tr}\left(\left(g+g^{\dagger}\right) \lambda^{2}\right)
$$

with $\lambda$ a generator of the group. Using diagonal generators, it is a straightforward exercise to see that for a maximum (minimum), the real parts of $g$ must all be positive (negative). All other solutions are saddle points. Thus all the maxima and minima are elements of the group center.

Specializing to $S U(3)$, there are four extremal classes. The single maximum of $\operatorname{Re} \operatorname{Tr} g$ occurs at the identity, two degenerate minima occur at the other two center elements $g=e^{ \pm 2 \pi i / 3}$, and a class of saddle point elements is represented by

$$
g=\left(\begin{array}{ccc}
-1 & 0 & 0 \\
0 & -1 & 0 \\
0 & 0 & 1
\end{array}\right)
$$




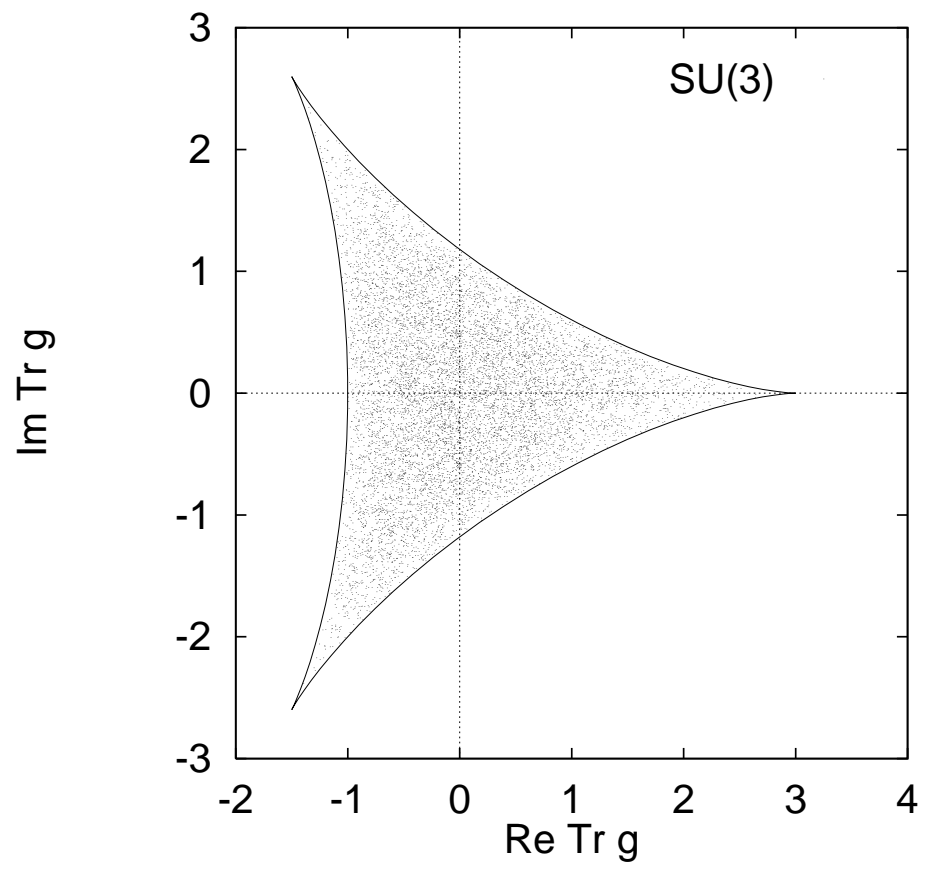

Fig. 5. Scatter plot of the traces of 10,000 randomly selected $S U(3)$ matrices. They are enclosed in the envelope discussed in the text. The spikes occur at the center elements of the group.

A complex curve that passes through all these extrema and encloses all values of $\operatorname{Tr} g$ is given by

$$
z=\operatorname{Tr} \exp (i \theta \lambda)
$$

with

$$
\lambda=\left(\begin{array}{ccc}
1 & 0 & 0 \\
0 & 1 & 0 \\
0 & 0 & -2
\end{array}\right)
$$

as theta runs from 0 to $2 \pi$. In Fig. (5) I plot this curve along with the traces of 10,000 randomly chosen $S U(3)$ matrices.

Using this basic structure of the $S U(3)$ group, it is now straightforward to see how the picture of first order phase transitions in the phase of the mass arises from the potential in Eq. (21). For $m>0$ the vacuum is the usual one with $g=I$. For a real but negative mass, the vacuum should lie at a minimum of $\operatorname{Re} \operatorname{Tr} g$, which occur in a degenerate pair at $g=e^{ \pm 2 \pi i / 3}$. Adding a small imaginary piece to $m$ breaks this degeneracy, showing the first order nature of the transition along the negative $m$ axis.

With a diagonal mass term $m=|m| e^{i \Theta / 3}$ with a general phase, the vacuum is always represented by one of these three extremal classes. For $\Theta=\pi$ there is a discontinuity, 
with the system jumping discontinuously from $g=I$ to $g=I e^{2 \pi i / 3}$. This is exactly the first order transition alluded to in the previous section. The three transition lines in the $\left(m_{1}, m_{2}\right)$ plane are physically equivalent since a phase change $m \rightarrow m e^{2 \pi i / 3}$ can be absorbed by taking $g \rightarrow g e^{-2 \pi i / 3}$. Later I will extend the above discussion to nondegenerate masses, providing a nice route between various numbers of flavors. 


\section{One flavor}

The one flavor situation, while not phenomenologically particularly relevant, is fascinating in its own right. In this case anomalies remove all chiral symmetries from the problem. No massless Goldstone Bosons are expected, and there is nothing to protect the quark mass from additive renormalization. Nevertheless, I have argued previously that there should be a non-trivial dependence on the phase of the mass. A large negative mass should be accompanied by a spontaneous breakdown of parity, as sketched in Fig. (3) .

A quick but dirty argument gives the expected picture. With only one flavor, there is only one light pseudo-scalar meson, which I call the $\eta$. Were it not for anomalies, conventional chiral symmetry arguments suggest the mass squared of this particle would go to zero linearly with the quark mass,

$$
m_{\eta}^{2} \sim m_{q}
$$

But, just as the $\eta^{\prime}$ gets mass from anomalies, a similar contribution should appear here; assume it is simply an additive constant

$$
m_{\eta}^{2} \sim m_{q}+C .
$$

Try to describe this model by an effective potential for the $\eta$ field. This should include the possibility for these particles to interact, suggesting something like

$$
V(\eta)=\frac{a m_{q}+C}{2} \eta^{2}+\lambda \eta^{4} .
$$

At $m_{q}=-C / a$ the effective mass of the eta goes negative. This should give a spontaneous breaking in the canonical manner, with the field acquiring an expectation value

$$
\langle\eta\rangle \sim\left\langle\bar{\psi} \gamma_{5} \psi\right\rangle \neq 0
$$

As this is an odd parity field, parity is spontaneously broken. In particular, odd numbers of physical mesons can be created, unlike in the unbroken theory where the number of mesons is preserved modulo 2 .

Note that this transition occurs at a negative quark mass, and nothing special happens at $m_{q}=0$. Of course the bare quark mass is a divergent quantity in need of renormalization. Without chiral symmetry, there is nothing to prevent an additive shift in this 
parameter. Nevertheless, with a cutoff in place, these qualitative arguments suggest it is only for negative quark mass that this parity violating phase transition will take place.

The anomaly involves processes that mix left and right handed quarks. This is exactly the role of a mass term, and thus the quark-antiquark pseudo-scalar bound state, the analogue of the $\eta^{\prime}$ meson, gains a mass. Now imagine making the quark masses slightly negative. This can cancel some of the mass generated by the anomaly and should reduce the mass of the meson. If the quarks become sufficiently negative in mass, one might decrease the bare mass of the meson to negative values. This gives the classic situation of spontaneous symmetry breaking and the meson field acquires an expectation value.

This argument is not at all rigorous. To lend more credence to this qualitative picture, note that a similar phenomenon occurs in two dimensional electrodynamics. The Schwinger model is exactly solvable at zero bare mass, with the spectrum being a free massive Boson. However for negative bare mass qualitative semi-classical arguments indicate the same structure as discussed in the previous paragraph, with a spontaneous generation of a parity violating background electric field (Creutz, 1995b). Under the Bosonizaton process, the quark mass term corresponds to a sinusoidal term in the effective potential for the scalar field

$$
m \bar{\psi} \psi \leftrightarrow m \cos (2 \sqrt{\pi} \eta)
$$

Regularization and normal ordering are required for a proper definition but are not important here (Coleman, 1976). Combining this with the photon mass from the anomaly suggests an effective potential for the $\eta$ field of form

$$
V(\eta) \sim \frac{e^{2}}{2 \pi} \eta^{2}-m \cos (2 \sqrt{\pi} \eta) .
$$

For small positive $m$, the second term shifts the Boson mass and introduces a four meson coupling, making the theory no longer free. If the mass is negative and large enough, the cosine term can dominate the behavior around small $\eta$, making the perturbative vacuum unstable. The Bosonization process relates $\bar{\psi} \gamma_{5} \psi$ with $\sin (2 \sqrt{\pi} \eta)$; thus, when $\eta$ gains an expectation value, so does the the pseudo-scalar density. Since the scalar field represents the electric field, this symmetry breaking represents the spontaneous generation of a background field. As discussed by Coleman (1976), this corresponds to a non-trivial topological term in the action, usually referred to as $\Theta$.

A third way to understand the one flavor behavior is to consider a larger number of flavors and give all but one large masses. In (Creutz, 1995a) this procedure was done 
starting with two flavors. That case required some additional assumptions regarding the spectrum; these assumptions become less important for larger symmetry groups. Thus I consider the three flavor case, and give two quarks a larger mass than the third.

I begin with the effective three flavor Lagrangian of the previous section. With two quarks of mass $M$ and one of mass $m$, consider the potential

$$
V(g) \propto-\operatorname{Re} \operatorname{Tr}\left\{g\left(\begin{array}{ccc}
m & 0 & 0 \\
0 & M & 0 \\
0 & 0 & M
\end{array}\right)\right\} .
$$

It is convenient to break this into two terms

$$
V(g) \propto-\frac{M+m}{2} \operatorname{Re} \operatorname{Tr}(g)+\frac{M-m}{2} \operatorname{Re} \operatorname{Tr}(g h)
$$

where

$$
h=\left(\begin{array}{ccc}
1 & 0 & 0 \\
0 & -1 & 0 \\
0 & 0 & -1
\end{array}\right)
$$

The minimum of the first term in Eq. (35) was worked out in the previous section; when $M+m$ is positive this occurs at the identity element. For the second term, note that I have written the factor of $g$ as an $S U(3)$ group element. Via the previous discussions, the extrema of this term occur when the product $g h$ is in the group center. For the case $M-m$ positive, there is a degenerate pair of minima occurring at

$$
g=e^{ \pm 2 \pi i / 3} h
$$

Eq. (35) has two competing terms, one having a unique minimum and the other having two degenerate ground states. For the degenerate case with $M=m$, only the first term is present and the vacuum is unique. However when $m=-M$ only the second term is present with its corresponding pair of degenerate vacuua. Between these points there is a critical value $m_{c}$ where the situation shifts between a unique and a doubly degenerate vacuum.

To determine the critical mass, consider matrices of form

$$
g=\exp \left\{i \phi\left(\begin{array}{ccc}
-2 & 0 & 0 \\
0 & 1 & 0 \\
0 & 0 & 1
\end{array}\right)\right\} \text {. }
$$

For these the potential is

$$
V(\phi) \propto-m \cos (2 \phi)-2 M \cos (\phi)
$$


The extremum at $\phi=0$ changes from a minimum to a maximum at $m=-M / 2$, the desired critical point. As discussed at the beginning of this section, it occurs at a negative value of $m$. The only dimensional scale present is $M$, to which the result must be proportional. This analysis immediately generalizes to larger groups: for $N_{f}$ flavors $m_{c}=\frac{-1}{N_{f}-1}$.

This discussion suggests that a similar phenomenon should occur with one flavor of Wilson Fermion. Here the bare mass is controlled by the hopping parameter. As the hopping parameter increases, the Fermion mass decreases. In the plane of the gauge coupling and hopping parameter, a critical line should mark where the above parity breaking begins. In the lattice context the possibility of such a phase was mentioned briefly by Smit (1980), and extensively discussed by Aoki (1989) and Aoki and Gocksch (1992). The latter papers also made some rather dramatic predictions for the breaking of both parity and flavor symmetries when more quark species are present. I will return to this phenomenon in section XII. 


\section{Two flavors}

The two flavor case can also be obtained as a limit from three flavors. However, since $S U(2)$ is a simple $S_{3}$ sphere, it is a somewhat more intuitive manifold. Here several simplifications make some features of the physics particularly clear. In this section for variety I will switch to a "linear" realization of the effective chiral Lagrangian. The discussion is closely based on (Creutz, 1995a).

I begin by defining eight fields around which the discussion revolves

$$
\begin{aligned}
\sigma & =c \bar{\psi} \psi \\
\vec{\pi} & =i c \bar{\psi} \gamma_{5} \vec{\tau} \psi \\
\eta & =i c \bar{\psi} \gamma_{5} \psi \\
\vec{\delta} & =c \bar{\psi} \vec{\tau} \psi
\end{aligned}
$$

The Fermion $\psi$ has two isospin components, for which $\vec{\tau}$ represents the standard Pauli matrices. The factor $c$ is inserted to give the fields their usual dimensions. Its value is not particularly relevant to the qualitative discussion that follows, but one convention is take $c=F_{\pi} /|\langle\bar{\psi} \psi\rangle|$ where $F_{\pi}$ is the pion decay constant and the condensate is in the standard æther.

Corresponding to each of these fields is a physical spectrum. In some cases this is dominated by a known particle. These include the familiar triplet of pions around 140 $\mathrm{MeV}$ and the eta at $547 \mathrm{MeV}$. The others are not quite so clean, with a candidate for the isoscalar $\sigma$ being the $f_{0}(980)$ and for the isovector $\delta$ being the $a_{0}(980)$. I will only use that the lightest particle in the $\delta$ channel appears to be heavier than the $\eta$.

Now consider an effective potential $V(\sigma, \vec{\pi}, \eta, \vec{\delta})$ for these fields. I first consider the theory with vanishing quark masses. In the continuum limit, the strong coupling constant is absorbed via the phenomenon of dimensional transmutation (Coleman and Weinberg, 1973), and all dimensionless quantities are in principle determined. In the full theory with the quark masses restored, the only parameters should be those masses and $\Theta$.

For the massless theory many of the chiral symmetries become exact. Because of the anomaly, the chiral transformation of Eq. (15), which mixes the $\sigma$ and $\eta$ fields, is not a good symmetry. However flavored axial rotations should be valid. For example, the rotation

$$
\psi \longrightarrow e^{i \gamma_{5} \tau_{3} \phi / 2} \psi
$$


which mixes $\sigma$ with $\pi_{3}$

$$
\begin{array}{r}
\sigma \longrightarrow \cos (\phi) \sigma+\sin (\phi) \pi_{3} \\
\pi_{3} \longrightarrow \cos (\phi) \pi_{3}-\sin (\phi) \sigma
\end{array}
$$

should be a good symmetry. This transformation also mixes $\eta$ with $\delta_{3}$

$$
\begin{gathered}
\eta \longrightarrow \cos (\phi) \eta+\sin (\phi) \delta_{3} \\
\delta_{3} \longrightarrow \cos (\phi) \delta_{3}-\sin (\phi) \eta
\end{gathered}
$$

For the massless theory, the effective potential is invariant under such rotations. In this two flavor case, the consequences can be compactly expressed by going to a vector notation. Define the four component objects $\Sigma=(\sigma, \vec{\pi})$ and $\Delta=(\eta, \vec{\delta})$. The effective potential is a function only of invariants constructed from these vectors. A complete set of invariants is $\left\{\Sigma^{2}, \Delta^{2}, \Sigma \cdot \Delta\right\}$. This separation into two sets of fields is special to the two flavor case, but makes the behavior of the theory particularly transparent.

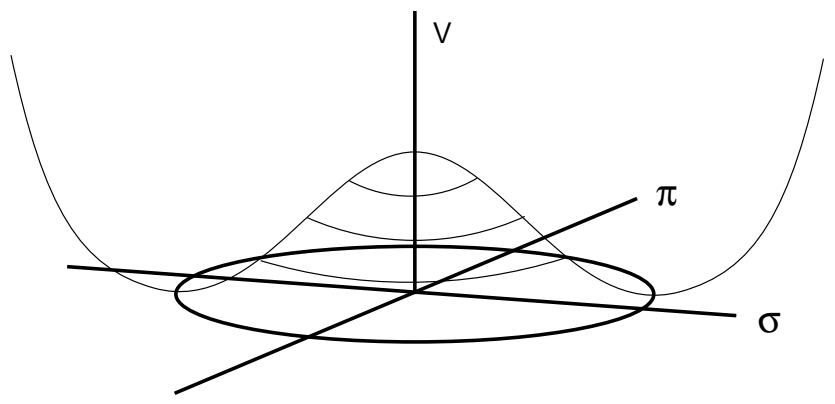

Fig. 6. The "sombrero" potential representing the chiral limit of massless quarks. Possible distortions of the circle representing the bottom of this potential are the main subject of this section.

I now use the experimental fact that chiral symmetry is spontaneously broken. The minimum of the effective potential should not occur for all fields having vanishing expectation. Assuming that parity and flavor are good symmetries of the strong interactions, the expectation value of the fields can be chosen in the $\sigma$ direction. Temporarily ignoring the fields $\Delta$, the potential should have the canonical "sombrero" shape, as stereotyped by the form

$$
V=\lambda\left(\Sigma^{2}-v^{2}\right)^{2}=\lambda\left(\sigma^{2}+\vec{\pi}^{2}-v^{2}\right)^{2}
$$

Here $v$ is the magnitude of the æther expectation value for $\sigma$, and $\lambda$ is a coupling strength related to the $\sigma$ mass. The normalization convention mentioned below Eq. (40) would 
have $v=F_{\pi} / 2$. I sketch the generic structure of the potential in Fig. (6) . This gives the standard picture of pions as Goldstone Bosons associated with fields oscillating along degenerate minima.

Now consider the influence of the fields $\Delta$ on this potential. Taking small fields, I expand the potential about vanishing $\Delta$

$$
V=\lambda\left(\Sigma^{2}-v^{2}\right)^{2}+\alpha \Delta^{2}-\beta(\Sigma \cdot \Delta)^{2}+\ldots
$$

Being odd under parity, $\Sigma \cdot \Delta$ appears quadratically.

The terms proportional to $\alpha$ and $\beta$ generate masses for the $\eta$ and $\delta$ particles. Since $\Delta^{2}=\eta^{2}+\vec{\delta}^{2}$, the $\alpha$ term contributes equally to each. Substituting $\Sigma \sim(v, \overrightarrow{0})$ gives $(\Sigma \cdot \Delta)^{2} \sim v^{2} \eta^{2}$; thus, the $\beta$ term breaks the $\eta-\vec{\delta}$ degeneracy. Here is where the observation that the $\eta$ is lighter than the $\delta$ comes into play; I have written a minus sign in Eq. (45), thus making the expected sign of $\beta$ positive.

Next I turn on the Fermion masses. I consider small masses, and assume they appear as a linear perturbation of the effective potential

$$
V \longrightarrow V-\left(M_{1} \cdot \Sigma+M_{2} \cdot \Delta\right) / c
$$

Here the four-component objects $M_{1,2}$ represent the possible mass terms. The normalization constant $c$ appears in Eq. (40). The zeroth component of $M_{1}$ gives a conventional mass term proportional to $\bar{\psi} \psi$, contributing equally to both flavors. The mass splitting of the up and down quarks appears naturally in the third component of $M_{2}$, multiplying $\bar{\psi} \tau_{3} \psi$. The term $m_{2}$ of Eq. (14) occupies the zeroth component of $M_{2}$.

The chiral symmetries of the problem require that physics only depends on invariants. For these I can take $M_{1}^{2}, M_{2}^{2}$, and $M_{1} \cdot M_{2}$. That there are three parameters is reassuring; there are the quark masses $\left(m_{u}, m_{d}\right)$ and the $\mathrm{CP}$ violating parameter $\Theta$. The mapping between these parameterizations is non-linear, the conventional definitions giving

$$
\begin{aligned}
M_{1}^{2} & =\frac{1}{4}\left(m_{u}^{2}+m_{d}^{2}\right)+\frac{1}{2} m_{u} m_{d} \cos (\Theta) \\
M_{2}^{2} & =\frac{1}{4}\left(m_{u}^{2}+m_{d}^{2}\right)-\frac{1}{2} m_{u} m_{d} \cos (\Theta) \\
M_{1} \cdot M_{2} & =\frac{1}{2} m_{u} m_{d} \sin (\Theta) .
\end{aligned}
$$

If one of the quark masses, say $m_{u}$, vanishes, then the $\Theta$ dependence drops out. This is sometimes proposed as a possible way to remove unwanted $\mathrm{CP}$ violation from the strong 
interactions; however, having a single quark mass vanish represents a fine tuning which is not obviously more compelling than simply tuning $\Theta$ to zero. Also, having $m_{u}=0$ appears to be phenomenologically untenable (Donoghue, Holstein, and Wyler, 1992; Leutwyler, 1990; Bijnens, Prades, de Rafael, 1995).

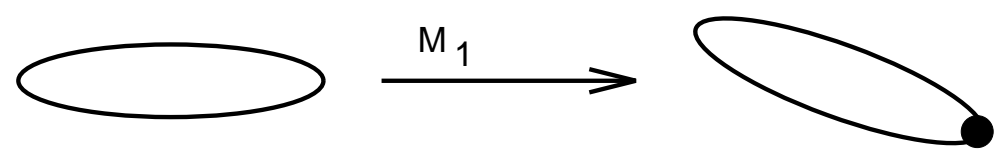

Fig. 7. The effect of $M_{1}$ is a tilting of the effective potential. The ellipse in this and the following figures represents the set of minima for the effective potential from Fig. (6) . The dot represents lowest energy state where the æether settles.

I now turn to a physical picture of what the two mass terms $M_{1}$ and $M_{2}$ do to the "Mexican hat" structure of the massless potential. For $M_{1}$ this is easy; its simply tilts the sombrero. This is sketched in Fig. (7) . The symmetry breaking is now driven in a particular direction, with the tilt selecting the direction for $\Sigma$ field to acquire its expectation value. This picture is well known, giving rise to standard relations such as the square of the pion mass being linearly proportional to the quark mass (Gell-Mann, Oakes, and Renner, 1968).

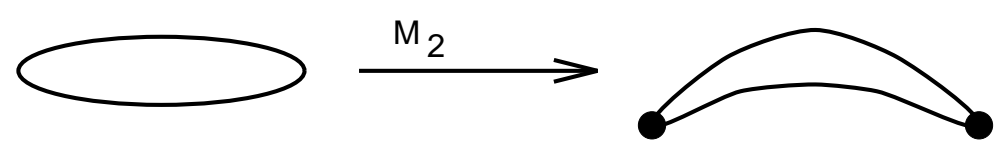

Fig. 8. The effect of $M_{2}$ is a warping of the effective potential. This is quadratic, with the dots representing the two places where the æther can settle.

The effect of $M_{2}$ is more subtle. This quantity has no direct coupling to the $\Sigma$ field; so, I must look to higher order. The $M_{2}$ term is a force pulling on the $\Delta$ field; it should give an expectation value proportional to the strength, $\langle\Delta\rangle \propto M_{2}$. Once $\Delta$ gains an expectation value, it then affects $\Sigma$ through the $\alpha$ and $\beta$ terms of the potential in Eq. (45). The $\alpha$ term is a function only of $\Sigma^{2}$, and, at least for small $M_{2}$, should not qualitatively change the structure of the symmetry breaking. On the other hand, the $\beta$ term will warp the shape of the sombrero. As this term is quadratic in $\Sigma \cdot \Delta$, this warping is quadratic. With $\beta$ positive, as suggested above, this favors an expectation value of $\Sigma$ lying along the vector $M_{2}$, but the sign of this expectation is undetermined. This effect is sketched in Fig. (8) . 
To summarize, the effect of $M_{1}$ is to tilt the Mexican hat, while the effect of $M_{2}$ is to install a quadratic warping. The three parameters are the amount of tilt, the amount of warping, and, finally, the relative angle between these effects. To better understand the interplay of these various phenomena, I now consider two specific situations in more detail.

First consider $M_{1}$ and $M_{2}$ parallel in the four vector sense. This is the situation with two mass terms of Eq. (14) and no explicit breaking of flavor symmetry. Specifically, I take $M_{1}=\left(m_{1}, \overrightarrow{0}\right)$ and $M_{2}=\left(m_{2}, \overrightarrow{0}\right)$. In this case the warping and the tilting are along the same axis.

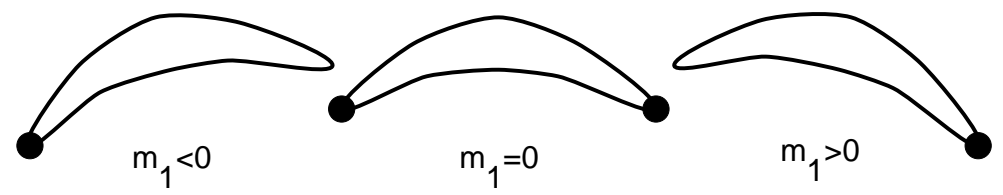

Fig. 9. Varying $m_{1}$ at fixed $m_{2}$. A first-order phase transition is expected at $m_{1}=0$. This corresponds to $\Theta=\pi$. The dots represent possible vacuum states.

Suppose I consider $m_{2}$ at some non-vanishing fixed value, and study the state of the æther as $m_{1}$ is varied. The $m_{2}$ term has warped the sombrero, but if $m_{1}$ is large enough, the potential will have a unique minimum in the direction of this pull. As $m_{1}$ is reduced in magnitude, the tilt decreases, and eventually the warping generates a second local minimum in the opposite $\sigma$ direction. As $m_{1}$ passes through zero, this second minimum becomes the lower of the two, and a phase transition occurs exactly at $m_{1}=0$. The transition is first order with the expectation of $\sigma$ jumping discontinuously. This situation is sketched in Fig. (9). From Eq. (47), the transition occurs at $m_{u}=m_{d}$ and $\Theta=\pi$.

As $m_{2}$ decreases, so does the warping, reducing the barrier between the two minima. This makes the transition softer. A small further perturbation in, say, the $\pi_{3}$ direction, will tilt the sombrero a bit to the side. If the warping is small enough, the field can then roll around the preferred side of the hat, thus opening a gap separating the positive $m_{2}$ phase transition line from that at negative $m_{2}$. In this way sufficient flavor breaking can remove the phase transition at $\Theta=\pi$ for small masses.

I now turn to a situation where $M_{1}$ and $M_{2}$ are orthogonal. To be specific, take $M_{1}=\left(m_{1}, \overrightarrow{0}\right)$ and $M_{2}=(0,0,0, \delta m)$, which physically represents a flavor symmetric mass term $m_{1}=\left(m_{u}+m_{d}\right) / 2$ combined with a flavor breaking $\delta m=\left(m_{u}-m_{d}\right) / 2$. Now $M_{2}$ warps the sombrero downwards in the $\pm \pi_{3}$ direction. A large $m_{1}$ would overcome this warping, still giving an æther with only $\sigma$ having an expectation value. However, 

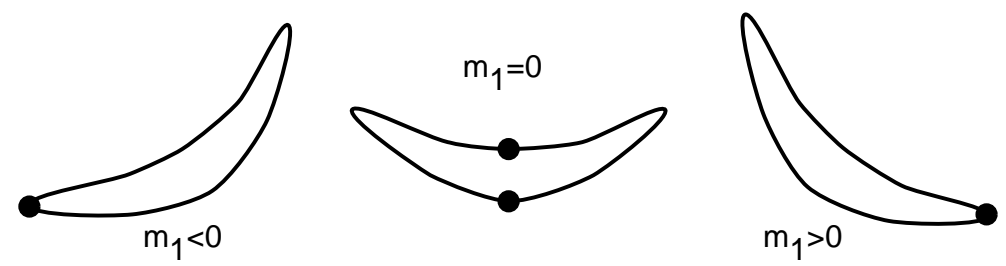

Fig. 10. Varying $m_{1}$ at fixed quark mass splitting. A second order phase transition occurs when the tilting is reduced sufficiently for a spontaneous expectation of $\pi_{3}$ to develop. The dots represent places where the æether can settle.

as $m_{1}$ decreases in magnitude with a fixed $\delta m$, there eventually comes a point where the warping dominates the tilting. A new symmetry breaking should occur, with $\pi_{3}$ acquiring an expectation value. This is sketched in Fig. (10). As $\pi_{3}$ is a CP odd operator, this is a spontaneous breaking of CP.

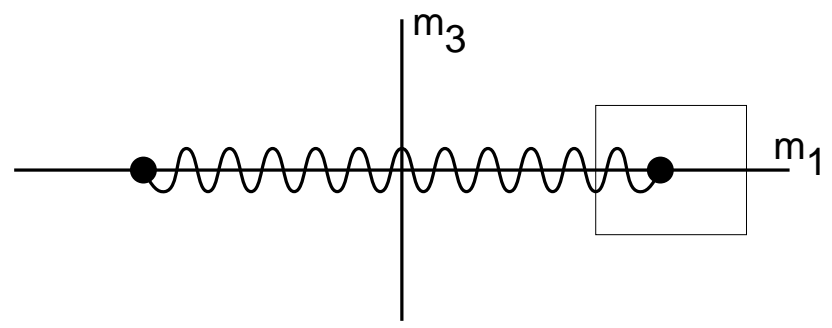

Fig. 11. The $\left(m_{1}, m_{3}\right)$ phase diagram for unequal mass quarks. The wavy line represents a first-order phase transition ending at the second order dots. The light box on the right shows how the one flavor case is extracted.

To make this into a proper two dimensional phase diagram, I add an $m_{3} \pi_{3}$ piece to the potential. This effectively twists $M_{1}$ away from being exactly perpendicular to $M_{2}$. This explicitly breaks CP and should remove the transition, just as an applied field removes the phase transition in the Ising model. We thus have a phase diagram in the $\left(m_{1}, m_{3}\right)$ plane with a first-order transition connecting two symmetrically separated points on the $m_{1}$ axis. This is sketched in Fig. (11) .

Physically, the endpoints of this transition line are associated with the points where the respective quark masses vanish. The phase transition occurs when the two flavors have masses of opposite sign. Simultaneously flipping the signs of both quark masses can always be undone by a flavored chiral rotation, say about the $\pi_{3}$ axis, and thus is a good symmetry of the theory. 
Taking one of the flavors to infinite mass provides another way to understand the one flavor situation discussed in the previous section. As sketched in Fig. (11), this represents looking only at the vicinity of one endpoint of the transition line. In terms of the light species, this transition represents a spontaneous breaking of $\mathrm{CP}$ with a non-vanishing expectation for $i \bar{\psi} \gamma_{5} \psi$. 


\section{Wilson Fermions for complex mass}

I now discuss the suggested implications of these ideas for Wilson's lattice Fermions. The picture is rather qualitative, and, as the next section will show, is not correct in fine details. Nevertheless, I hope to convince you that the doublers, although raised to high masses in the Wilson approach, are responsible for a rather complex phase structure.

Generalizing Eq. (10) to complex mass leads to the action

$$
\begin{aligned}
& S\left(K, r, m_{1}, m_{2}\right)= \\
& \sum_{j, \mu} K\left(\bar{\psi}_{j}\left(i \gamma_{\mu}+r\right) \psi_{j+e_{\mu}}+\bar{\psi}_{j+e_{\mu}}\left(-i \gamma_{\mu}+r\right) \psi_{j}\right)+ \\
& \sum_{j}\left(m_{1} \bar{\psi}_{j} \psi_{j}+i m_{2} \bar{\psi}_{j} \gamma_{5} \psi_{j}\right) .
\end{aligned}
$$

Here $j$ labels the sites of a four dimensional hyper-cubic lattice, $\mu$ runs over the space-time directions, and $e_{\mu}$ is the unit vector in the $\mu$ 'th direction. I have scaled out all factors of the lattice spacing. The parameter $K$ is called the hopping parameter, and $r$ controls the strength of the so called "Wilson term," which separates off the famous doublers. I have also added $m_{2}$ type mass term as in Eq. (14) to connect with my earlier discussion.

Being quadratic with only nearest neighbor couplings, the free spectrum is easily found by Fourier transformation. Conventionally, a massless Fermion appears at $m_{1}=8 \mathrm{Kr}$, but there are other values of the parameters where this original particle is massive while other doublers from the naive theory become massless. At $m_{1}=-8 K r$ one such species does so, at each of $m_{1}= \pm 4 K r$ there are four massless doublers, and at $m_{1}=0 \mathrm{I}$ find the remaining 6 of the total 16 species present in the naive theory.

A natural conjecture is that these various species should be thought of as flavors. When the gauge fields are turned on, the full chiral structure should be a generalization of the discussion in the previous sections. Thus somewhere near $m_{1}=8 K r$ I expect a firstorder transition to end, much as is indicated in Fig. (3) . This may join with numerous other transitions at the intermediate values of $m_{1}$, all of which then finally merge to give a single first-order transition line ending near $m_{1}=-8 K r$. The situation near 0 and $\pm 4 K r$ involves larger numbers of light flavors, giving more transition lines. One possible way the lines could join up is shown in part $a$ of Fig. (12) .

For two flavors of Wilson Fermions, near to the singularity at $8 K r$ one should obtain a picture similar to Fig. (4) . However, further away these lines can curve and eventually end in the structure at the other doubling points. One possible picture is sketched in part $b$ of 

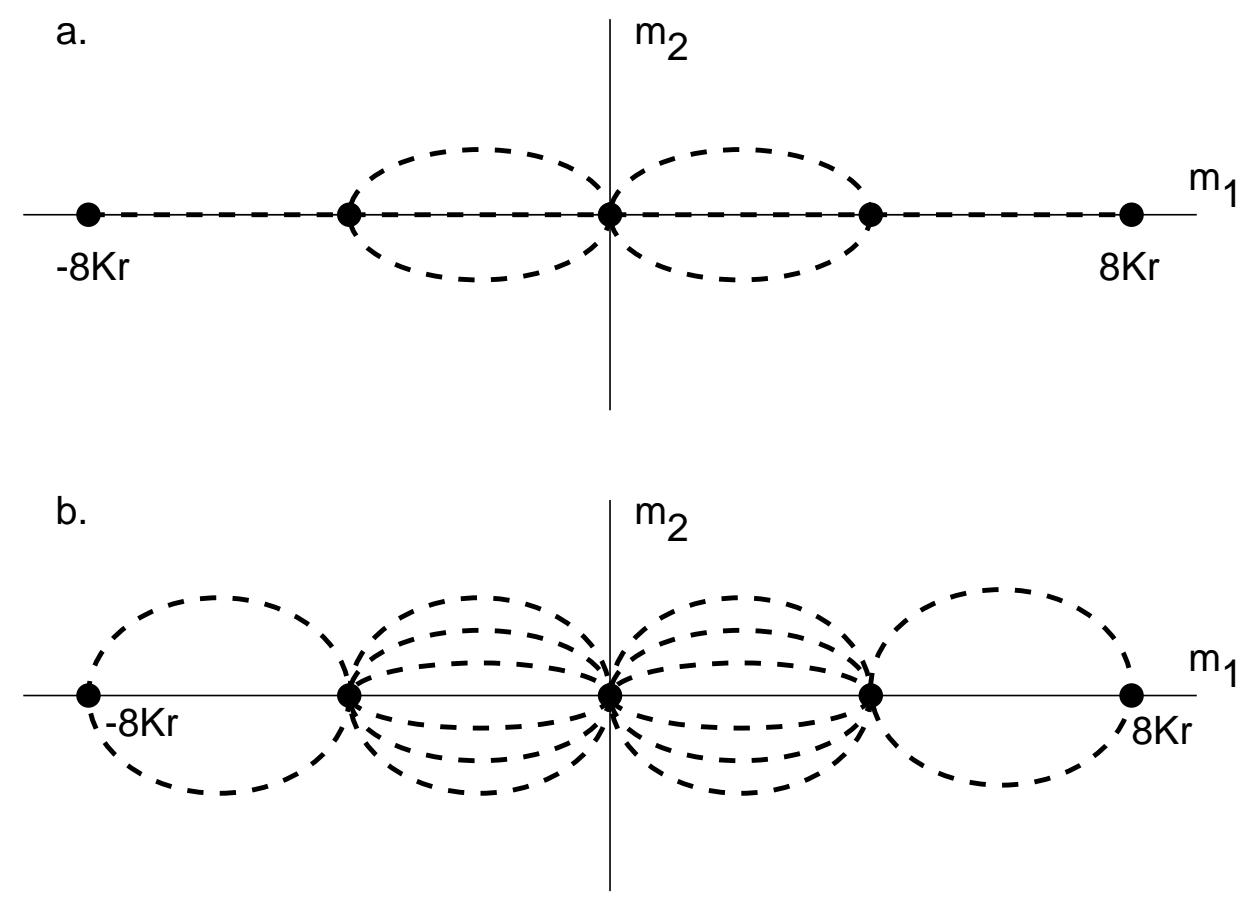

Fig. 12. Possible phase diagrams for lattice gauge theory with Wilson Fermions. The dashed lines represent first-order phase transitions and the dots represent points where massless excitations should exist. Parts (a) and (b) are for the one and two flavor cases, respectively.

Fig. (12) . While this is likely the qualitative behavior near the continuum limit, at more modest lattice spacings the situation is likely somewhat more complicated. Indeed, since the Wilson term violates chiral symmetry, the second order phase transition at vanishing mass is probably not robust. The resulting lattice artifacts are quite fascinating, and form the topic of the next section. 


\section{The Aoki phase}

In this section I probe further into the rather rich phase structure expected for lattice gauge theory with Wilson Fermions, paying particular attention to what happens for hopping parameters very near the critical value. This behavior was predicted some time ago (Aoki, 1989; Aoki and Gocksch, 1992; Aoki, Ukawa and Umemura, 1996) in attempts to understand the meaning of the phase transition that occurs with Wilson Fermions when the pseudo-scalar masses go to zero. As there is no chiral symmetry when the cutoff is in place, the "continuum" argument that this represents a spontaneous breaking of chiral symmetry is inadequate. This picture involved a spontaneous breaking of parity for one flavor, and a breaking of both flavor and parity symmetries for more species. The one flavor prediction is exactly the spontaneous breaking of parity discussed in the section on one flavor. However, for multiple flavors, the situation is a bit more complicated: the predicted spontaneous breaking of flavor symmetry gives additional Goldstone Bosons. In Aoki's picture with two flavors, at the critical hopping there are three massless particles, one being associated with the phase transition and the other two being the Goldstone Bosons of the flavor breaking.

Here I will argue that this picture is also easily understood in terms of effective Lagrangians. Indeed, the result appears to be inevitable, although variant actions could change the transition to first order. This discussion is based on Creutz (1997) and a similar treatment appears in Sharpe and Singleton (1998). While I frame the discussion about the Wilson-Fermion approach, the phenomenon appears to be generic in any approach that introduces chiral-symmetry breaking terms at finite cutoff.

I begin by reiterating that the only parameters of the strong interactions are the quark masses. I implicitly include here the strong $\mathrm{CP}$ violating parameter $\Theta$, as this can generally be rotated into the mass matrix. The strong coupling is not a parameter, having been absorbed into the units of measurement via the phenomenon of dimensional transmutation (Coleman and Weinberg, 1973).

For this section I take degenerate quarks at $\Theta=0$; thus, I consider only a single mass parameter $m$. I discuss only the two flavor case, as this will make some of the chiral symmetry issues simpler. In addition, as I am discussing the theory on the lattice, I introduce the lattice spacing $a$ as a second parameter.

On the lattice with Wilson Fermions, the physical parameters $(m, a)$ are usually replaced with $\beta$, representing the inverse bare coupling squared, and the Fermion hopping 
parameter $K$. I ignore the Wilson parameter $r$, which can be regarded as being fixed at unity, and set $M=1$, the coefficient of diagonal piece of the action from Eq. (10). The mapping between $(m, a)$ and $(\beta, K)$ is non-linear, well known, and not at issue here. Note that in considering the structure of the theory in either of these sets of variables, I am inherently talking about finite lattice spacing $a$. This entire section is about lattice artifacts.

I wish to develop a qualitative phase diagram for the $(\beta, K)$ plane. The $\beta$ axis with $K=0$ represents the pure gauge theory of glue-balls. This is expected to be confining without any singularities at finite $\beta$. The line of varying $K$ with $\beta=\infty$ represents free Wilson Fermions (Wilson, 1977). Here, with conventional normalizations, the point $K=\frac{1}{8}$ is where the mass gap vanishes and a massless Fermion should appear. The full interacting continuum limit should be obtained by approaching this point from the interior of the $(\beta, K)$ plane.

While receiving the most attention, the point $K=\frac{1}{8}$ is not the only place where free Wilson Fermions lose their mass gap. As discussed earlier, at $K=\frac{1}{4}$ four doubler species become massless. Also formally at $K=\infty$ six doublers loose their mass. (Actually, a more natural variable is $\frac{1}{K}$.) The remaining doublers become light at negative $K$.

The $K$ axis at vanishing $\beta$ also has a critical point where the confining spectrum appears to develop massless states. Strong coupling arguments as well as numerical experiments place this point near $K=\frac{1}{4}$, but this is probably not exact. The conventional picture connects this point to $\left(\beta=\infty, K=\frac{1}{8}\right)$ by a phase transition line representing the lattice version of the chiral limit.

Now I move ever so slightly inside the $(\beta, K)$ plane from the point $\left(\infty, \frac{1}{8}\right)$. This should lead from free quarks to a confining theory, with mesons, baryons, and glue-balls being the physical states. Furthermore, massless quarks should give chiral symmetry. Considering here the two flavor case, this symmetry is nicely exemplified in the "sigma" model, with three pion fields and one sigma field rotating amongst themselves. Here I only need these four fields from Section X

$$
\begin{aligned}
\sigma & =c \bar{\psi} \psi \\
\vec{\pi} & =i c \bar{\psi} \gamma_{5} \vec{\tau} \psi .
\end{aligned}
$$

As before, I consider constructing an effective potential. For massless quarks this is expected to have the canonical sombrero shape stereotyped by

$$
V \sim \lambda\left(\sigma^{2}+\vec{\pi}^{2}-v^{2}\right)^{2}
$$


and illustrated schematically in Fig. (6) . The normal æther is taken with an expectation value for the sigma field $\langle\sigma\rangle \sim v$. The physical pions are massless Goldstone Bosons associated with slow fluctuations of the æther along the degenerate minima of this potential.

As I move up or down in $K$ from the massless case near $\frac{1}{8}$, this effective potential will tilt in the standard way, with the sign of $\langle\sigma\rangle$ being appropriately determined. The role of the quark mass is played by the distance from the critical hopping, $m_{q} \sim K_{c}-K$ with $K_{c} \sim \frac{1}{8}$. At the chiral point there occurs a phase transition, with the sign of $\langle\sigma\rangle$ jumping discontinuously. At the transition point massless Goldstone pions represent the spontaneous symmetry breaking. With an even number of flavors the basic physics on each side of the transition is the same; the sign of the mass term is a convention reversible via a non-singlet chiral rotation. (For an odd number of flavors the sign of the mass is significant because the required rotation involves the $U(1)$ anomaly and is not a good symmetry. This is discussed in some detail in earlier sections.)

A similar picture should also occur near $K=\frac{1}{4}$, representing the point where a subset of the Fermion doublers become massless. Thus another phase transition should enter the diagram at $K=\frac{1}{4}$. Similar lines will enter at negative $K$ and further complexity occurs at $K=\infty$. For simplicity, let me concentrate only on the lines entering from large $\beta$ at $K=\frac{1}{8}$ and $\frac{1}{4}$.

Now I delve a bit deeper into the $(\beta, K)$ plane. The next observation is that the Wilson term is explicitly not chiral invariant. This should damage the beautiful symmetry of the sombrero. The first effect expected is a general tilting of the potential. This represents an additive renormalization of the Fermion mass, and appears as a beta dependent motion of the critical hopping away from $\frac{1}{8}$. Define $K_{c}(\beta)$ as the first singularity in the phase diagram for increasing $K$ at given $\beta$. This gives a curve which presumably starts near $K=\frac{1}{4}$ at $\beta=0$ and ends up at $\frac{1}{8}$ for infinite $\beta$.

Up to this point this is standard lore. Now I continue to delve yet further away from the continuum chiral point at $(\beta, K)=\left(\infty, \frac{1}{8}\right)$. Then I expect the chiral symmetry breaking of the Wilson term to increase in importance and do more than simply tilt the Mexican hat. I'm not sure to what extent a multi-pole analysis of this breaking makes sense, but let me presume that the next effect is a quadratic warping of the sombrero, i.e. a term something like $\alpha \sigma^{2}$ appearing in the effective sigma model potential. This warping cannot be removed by a simple mass renormalization.

There are two possibilities. This warping could be upward or downward in the $\sigma$ 
direction. Indeed, which possibility occurs might depend on the value of $\beta$. Consider first the case where the warping is downward, stabilizing the sigma direction for the æether. At the first order chiral transition, this distortion gives the pions a small mass. The transition then occurs without a diverging correlation length. As before, the condensate $\langle\sigma\rangle$ jumps discontinuously, changing its sign. The conventional approach of extrapolating the pion mass to zero from measurements at smaller hopping parameter will no longer yield the correct critical line. The effect of this warping on the potential is illustrated in Fig. (9) from section X. There is a single first order phase transition without any divergent correlation lengths.

The second possibility is for the warping to be in the opposite direction, destabilizing the $\sigma$ direction. In this case we expect two distinct phase transitions to occur as $K$ passes through the critical region. Small hoppings give a tilted potential with $\sigma$ having a positive expectation. As $K$ increases, this tilting will eventually be insufficient to overcome the destabilizing influence of the warping. At a critical point, most likely second order, it will become energetically favorable for the pion field to acquire an expectation value, such a case being stabilized by the upward warping in the sigma direction. As $K$ continues to increase, a second transition should appear where the tilting of the potential is again sufficiently strong to give only sigma an expectation, but now in the negative direction. The effect of this upward warping on the effective potential is equivalent to the situation illustrated in Fig. (10) .

Thus the critical line splits into two, with a rather interesting phase between. This phase has a non-vanishing expectation value for the pion field. As the latter carries flavor and odd parity, both are spontaneously broken. Furthermore, since flavor is still an exact continuous global symmetry, when it is broken Goldstone Bosons will appear. In this two flavor case, there are precisely two such massless excitations. If the transitions are indeed second order, a third massless particle appears on the transition lines, and these three particles are the remnants of the pions from the continuum theory. This "Aoki" phase should be "pinched" between the two transitions, and become of less importance as $\beta$ increases and the continuum limit is approached. Whether the phase might be squeezed out at a finite $\beta$ to the first order situation, or whether it only disappears in the infinite $\beta$ limit is a dynamical question.

A similar critical line splitting to give a broken flavor phase may also enter the phase diagram from $(\beta, K)=\left(\infty, \frac{1}{4}\right)$, representing the first set of doublers. Evidence from toy 


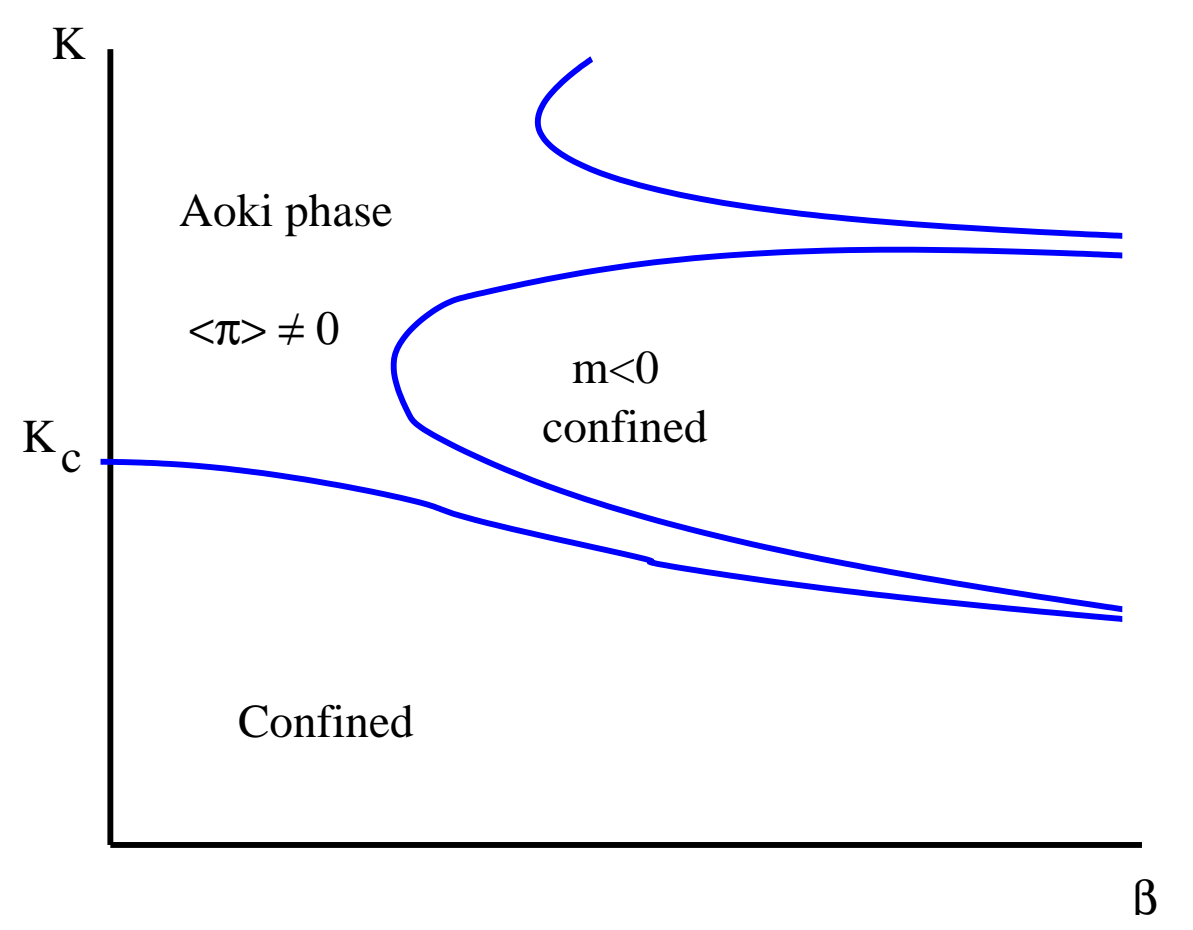

Fig. 13. The conjectured $(\beta, K)$ phase diagram. In the Aoki phase flavor and parity are spontaneously broken via the pion field acquiring an expectation value.

models (Aoki, Boetcher, and Gocksch, 1994; Bitar and Vranas, 1994a,b; Aoki, Ukawa and Umemura, 1996) is that after this line splits, the lower half joins up with the upper curve from the $(\beta, K)=\left(\infty, \frac{1}{8}\right)$ point. In these models, there appears to be only one broken parity phase at strong coupling.

Fig. (13) summarizes the picture. Small $\beta$ along with small $K$ gives the massive quark confined phase. Increasing $K$ brings one to the Aoki phase with spontaneous breaking of flavor and parity. As $\beta$ increases, the Aoki phase pinches down into either a narrow point or a single first order line, leading towards the free Fermion point at $(\beta, K)=\left(\infty, \frac{1}{8}\right)$.

This diagram is wonderfully complex, probably incomplete, and may take some time to map out. As a final reminder, this entire discussion is of lattice artifacts, and other lattice actions, perhaps including various "improvements," can look dramatically different. In particular, other actions may display the alternative option for a single first order transition instead of the two transitions surrounding the Aoki phase. 


\section{The Wess-Zumino term}

The domain-wall Fermion approach, toward which I am leading, uses an extra spacetime dimension. Earlier sections showed how effective Lagrangians give considerable insight into the physical consequences of anomalous processes. The idea that extra dimensions could be useful for related phenomena appeared some time ago in the context of these chiral Lagrangians. Indeed, with hindsight, the analogy between one term, called the Wess Zumino term, and domain-wall Fermions is striking (Creutz and Tytgat, 1996). In this section I give a pedagogical discussion of the former as a motivation for looking at extra dimensions.

In the previous two sections I used what is often called the "linear" approach to effective chiral Lagrangians. Here I return to the "non-linear" approach and repeat some of the equations used in the three flavor section. Consider quark fields $\psi^{a}$ with a flavor index $a$ running from one to the number of flavors $N_{f}$. Since the quark fields are expected to have a chiral condensate, the vacuum is not unique. Consider the general matrix valued quantity

$$
{ }_{g}\left\langle 0\left|\bar{\psi}_{L}^{a} \psi_{R}^{b}\right| 0\right\rangle_{g}=v g^{a b} .
$$

Here I pick $v$ to normalize things so that the vacuum is labeled by $g$, an element of the flavor group $S U\left(N_{f}\right)$. For the usual vacuum one would pick the identity element, but flavored chiral rotations allow turning to alternate degenerate states. A general chiral transformation is specified by two $S U(N)$ matrices $\left\{g_{L}, g_{R}\right\}$ and takes

$$
\psi_{L, R}^{a} \rightarrow \psi_{L, R}^{b} g_{L, R}^{b a} .
$$

Under this the effective field $g$ from Eq. (51) transforms as

$$
g \rightarrow g_{L}^{\dagger} g g_{R} .
$$

The basic chiral Lagrangian idea is to integrate out all other fields, generating an effective action for the above matrix $g$. This is expanded in powers of derivatives of the group-valued field. The lowest term

$$
S_{\text {eff }}=\frac{F_{\pi}^{2}}{4} \int(d g) \operatorname{Tr}\left(\partial_{\mu} g^{\dagger} \partial_{\mu} g\right)
$$

appeared in earlier sections. Chiral perturbation theory involves enumerating and elucidating the higher derivative couplings that can be added while preserving the chiral 
symmetries. As mentioned earlier, the constant $F_{\pi}$ has an experimental value $93 \mathrm{MeV}$. In terms of conventional fields, $g=\exp \left(i \pi \cdot \lambda / F_{\pi}\right)$, where the $n_{f}^{2}-1$ traceless matrices $\lambda$ generate $S U\left(n_{f}\right)$ and are normalized $\operatorname{Tr} \lambda^{\alpha} \lambda^{\beta}=2 \delta^{\alpha \beta}$. From this action, the equations of motion can be compactly written $\partial_{\mu} J_{L, \mu}^{\alpha}=0$, where the "left" current is

$$
J_{L, \mu}^{\alpha}=\frac{i F_{\pi}^{2}}{4} \operatorname{Tr}\left(\lambda^{\alpha}\left(\partial_{\mu} g\right) g^{\dagger}\right)
$$

Equivalently, one can work with "right" currents. There is a vast literature on possible higher derivative terms (Donoghue, Holstein, and Wyler, 1992; Leutwyler, 1990; Bijnens, Prades, and de Rafael, 1995).

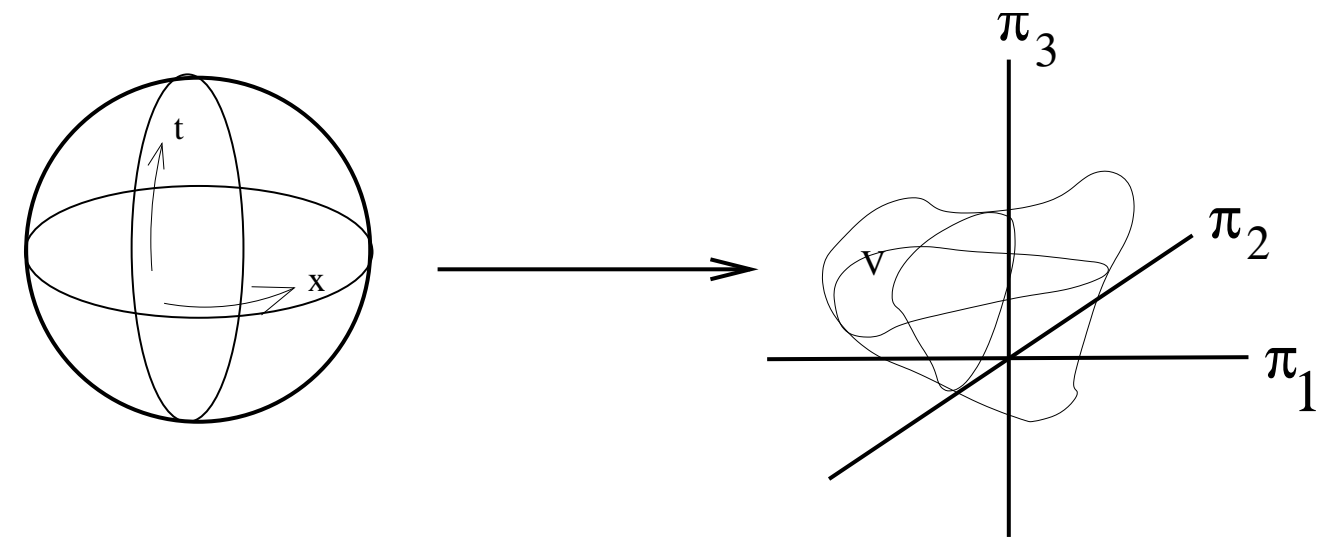

Fig. 14. Mapping two dimensional space-time into three dimensional field space.

The closed surface surrounds a volume which is invariant under chiral shifts.

For my purposes here, however, I concentrate on one particular term with a subtle topological significance (Wess and Zumino, 1971; Witten, 1983a,b, 1984). To understand this pictorially, it is convenient to work first with the two flavor case in two space-time dimensions. Then $g \in S U(2)$ represents three massless pion fields. In the path integral a field configuration is a mapping of space-time into field space. For convenience, imagine I have compactified space-time onto the surface of a two dimensional sphere. A general field configuration is a mapping of this two dimensional sphere into the three dimensional manifold of fields. Since the sphere is closed, this mapping will surround a closed region in field space. This is illustrated in Fig. (14) An interesting quantity to consider is the volume of this enclosed region. Indeed, lattice gauge theorists are intimately familiar with group invariant integration measures, and, since $g$ lies in a group, it is natural to use this measure to define this volume. Furthermore, the properties of the group measure insure that the 
volume is invariant under the chiral symmetry of Eq. (53) . Thus a possible interaction term that satisfies chiral symmetry is just this volume

$$
S_{I}=\lambda \int_{V} d g
$$

However, the group manifold $S U(2)$ is compact. This means that it is not easy to be sure what is "inside" the surface mapped out by the field. There is an ambiguity in defining this volume up to the volume of the entire group. A gradual deformation of the field can take a small volume and grow it to fill the group such that what was originally inside the volume now looks like it is outside. In short, after normalizing the group measure so that the total volume of the group is unity, $\int_{G} d g=1$, the interaction term is ambiguous up to an integer. For quantum mechanics this would not be important if the coupling $\lambda$ was a multiple of $2 \pi$. Then the quantity relevant to the path integral, $e^{i S}$, is unchanged when the action changes by $2 \pi$ times an integer. This quantization of a coupling constant is a field theoretical generalization of the quantization of magnetic monopole charge in electrodynamics (Witten 1983a,b, 1984).

But note that the expression for this interaction term in Eq. (56) is rather abstract. Remarkably, this term cannot be written as a simple space-time integral over the local fields and their derivatives. Such an expression would not have the ambiguity indicated in the previous paragraph. To obtain an explicit expression requires introducing an extra dimension, allowing continuation inside the volume indicated in Fig. (14) . For this let me pick an origin in the group; this could be the identity element, but I will be more general and pick some specific element $g_{0}$. Then introduce an extra dimension $s$, which for convenience I let run from 0 to 1 . I extend my field $g(x, t)$ to a three dimensional one $h(x, t, s) \in S U(2)$. I subject the extended field to two constraints

$$
\begin{aligned}
& h(x, t, 0)=g_{0} \\
& h(x, t, 1)=g(x, t) .
\end{aligned}
$$

With this extension, the dimension of space-time now matches the dimension of the field manifold. This enables a rather compact and elegant expression for the volume enclosed by the field

$$
\int_{V} d g=\frac{1}{24 \pi^{2}} \int_{V} d^{3} x \epsilon_{\mu \nu \rho} \operatorname{Tr}\left(h^{\dagger} \partial_{\mu} h h^{\dagger} \partial_{\nu} h h^{\dagger} \partial_{\rho} h\right) .
$$

To obtain the numerical factor of $\frac{1}{24 \pi^{2}}$, take a field configuration that covers the group once and require that the integral be unity. 
This entire discussion extends to higher dimensions with larger groups. The dimension of the group must exceed the dimension of space time, so four dimensions requires at least $S U(3)$. This allows mappings into the group space which are ambiguous due to the possibilities of non-trivial topology (Witten, 1983a,b, 1984). To obtain this term from an action requires extending $g(x)$ beyond a simple mapping of space-time into the group. As before, introduce an auxiliary variable $s$ to interpolate between the field $g(x)$ and some fixed group element $g_{0}$. Thus consider $h(x, s)$ satisfying $h(x, 1)=g(x)$ and $h(x, 0)=g_{0}$. This extension is not unique, but the equations of motion are independent of the chosen path. In analogy with Eq. (58), write

$$
\begin{aligned}
S & =\frac{F_{\pi}^{2}}{4} \int d^{4} x \operatorname{Tr}\left(\partial_{\mu} g \partial_{\mu} g^{\dagger}\right) \\
& +\frac{n_{c}}{240 \pi^{2}} \int d^{4} x \int_{0}^{1} d s \epsilon_{\alpha \beta \gamma \delta \rho} \operatorname{Tr} h_{\alpha} h_{\beta} h_{\gamma} h_{\delta} h_{\rho} .
\end{aligned}
$$

Here I define $h_{\alpha}=i\left(\partial_{\alpha} h\right) h^{\dagger}$ and regard $s$ as a fifth coordinate for the antisymmetric tensor.

For equations of motion, consider a small variation of $h(x, s)$. This changes the final integrand by a total divergence, which integrates to a surface term. Working with either spherical or toroidal boundary conditions in the space-time directions, this surface only involves the boundaries of the $s$ integration. When $s=0$, space-time derivatives acting on the constant matrix $g_{0}$ vanish. Writing the equations of motion in terms of a divergence free current gives

$$
\begin{aligned}
J_{L, \mu}^{\alpha} & =\frac{i F_{\pi}^{2}}{4} \operatorname{Tr}\left(\lambda^{\alpha}\left(\partial_{\mu} g\right) g^{\dagger}\right) \\
& +\frac{i n_{c}}{48 \pi^{2}} \epsilon_{\mu \nu \rho \sigma} \operatorname{Tr}\left(\lambda^{\alpha}\left(\partial_{\nu} g\right) g^{\dagger}\left(\partial_{\rho} g\right) g^{\dagger}\left(\partial_{\sigma} g\right) g^{\dagger}\right) .
\end{aligned}
$$

The last term in Eq. (59) represents a piece cut from the $S_{5}$ sphere appearing in the structure of $S U\left(n_{f}\right)$ for $n_{f} \geq 3$. The mapping of four dimensional space-time into the group surrounds this volume. Chiral rotations shift this region around, leaving its measure invariant. As emphasized by Witten (1983a,b, 1984), this term is ambiguous. Different extensions into the $s$ coordinate can modify the above five dimensional integral by an integer multiple of $480 \pi^{3}$. To have a well defined quantum theory, the action must be determined up to a multiple of $2 \pi$. Thus the quantization of $n_{c}$ to an integer, which Witten has argued represents the number of "colors."

Crucial here is the irrelevance of the starting group element $g_{0}$ at the lower end of the $s$ integration. An important issue is the difficulty of maintaining this condition if the 
chiral symmetry becomes local. As usual, this requires the introduction of gauge fields. Under the transformation $g(x) \rightarrow g_{L}^{\dagger}(x) g(x) g_{R}(x)$, derivatives of $g$ transform as

$$
\partial_{\mu} g \longrightarrow g_{L}^{\dagger}\left(\partial_{\mu} g-\partial_{\mu} g_{L} g_{L}^{\dagger} g+g \partial_{\mu} g_{R} g_{R}^{\dagger}\right) g_{R}
$$

To compensate, introduce left and right gauge fields transforming as

$$
\begin{aligned}
& A_{L, \mu} \quad \longrightarrow g_{L}^{\dagger} A_{L, \mu} g_{L}+i g_{L}^{\dagger} \partial_{\mu} g_{L} \\
& A_{R, \mu} \quad \longrightarrow g_{R}^{\dagger} A_{R, \mu} g_{R}+i g_{R}^{\dagger} \partial_{\mu} g_{R} .
\end{aligned}
$$

Then the combination

$$
D_{\mu} g=\partial_{\mu} g-i A_{L, \mu} g+i g A_{R, \mu}
$$

transforms nicely: $D_{\mu} g \rightarrow g_{L}^{\dagger} D_{\mu} g g_{R}$. The generalized minimal replacement $\partial_{\mu} g \rightarrow D_{\mu} g$ in $S_{0}$ gives a gauge invariant action.

A problem arises on coupling gauge fields to the Wess-Zumino term. This requires a prescription for the gauge transformation of the interpolated group element $h(x, s)$. Here I anticipate a striking analogy with the domain-wall approach to chiral Fermions. There an extra dimension is also introduced, with the Fermions being surface modes bound to a four dimensional interface. The usual method for adding gauge fields involves, first, not giving the gauge fields a dependence on the extra coordinate, and, second, forcing the component of the gauge field pointing in the extra dimension to vanish. In terms of a five dimensional gauge field, take $A_{\mu}(x, s)=A_{\mu}(x)$ and $A_{s}=0$ for both the left and right handed parts. Relaxing either of these would introduce unwanted degrees of freedom. The natural extension of the gauge transformation is to take $h(x, s) \rightarrow g_{L}^{\dagger}(x) h(x, s) g_{R}(x)$ with $g_{L, R}$ independent of $s$.

I now replace the derivatives in the Wess-Zumino term with covariant derivatives. This alone does not give equations of motion independent of the interpolation into the extra dimension. However, adding terms linear and quadratic in the gauge field strengths allows construction of a five dimensional Wess-Zumino term for which variations are again a total derivative. This gives

$$
S_{W Z}=\frac{n_{c}}{240 \pi^{2}} \int d^{4} x \int_{0}^{1} d s \Gamma
$$

where

$$
\begin{aligned}
\Gamma & =\Gamma_{0}+\frac{5 i}{2}\left(i \Gamma_{L}+i \Gamma_{R}\right. \\
& \left.-\Gamma_{L L}-\Gamma_{R R}-\alpha \Gamma_{L R}-(1-\alpha) \Gamma_{R L}\right),
\end{aligned}
$$


Here $\alpha$ is a free parameter and

$$
\begin{aligned}
& \Gamma_{0}=\epsilon_{\mu \nu \rho \lambda \sigma} \operatorname{Tr} \mathrm{D}_{\mu} \mathrm{hh}^{\dagger} \mathrm{D}_{\nu} \mathrm{hh}^{\dagger} \mathrm{D}_{\rho} \mathrm{hh}^{\dagger} \mathrm{D}_{\lambda} \mathrm{hh}^{\dagger} \mathrm{D}_{\sigma} \mathrm{hh}^{\dagger} \\
& \Gamma_{L}=\epsilon_{\mu \nu \rho \lambda \sigma} \operatorname{TrD}_{\mu} \mathrm{hh}^{\dagger} \mathrm{D}_{\nu} \mathrm{hh}^{\dagger} \mathrm{D}_{\rho} \mathrm{hh}^{\dagger} \mathrm{F}_{\mathrm{L}, \lambda \sigma} \\
& \Gamma_{R}=\epsilon_{\mu \nu \rho \lambda \sigma} \operatorname{TrD}_{\mu} \mathrm{hh}^{\dagger} \mathrm{D}_{\nu} \mathrm{hh}^{\dagger} \mathrm{D}_{\rho} \mathrm{hF}_{\mathrm{R}, \lambda \sigma} \mathrm{h}^{\dagger} \\
& \Gamma_{L L}=\epsilon_{\mu \nu \rho \lambda \sigma} \operatorname{TrD}_{\mu} \mathrm{hh}^{\dagger} \mathrm{F}_{\mathrm{L}, \nu \rho} \mathrm{F}_{\mathrm{L}, \lambda \sigma} \\
& \Gamma_{R R}=\epsilon_{\mu \nu \rho \lambda \sigma} \operatorname{TrD}_{\mu} \mathrm{hF}_{\mathrm{R}, \nu \rho} \mathrm{F}_{\mathrm{R}, \lambda \sigma} \mathrm{h}^{\dagger} \\
& \Gamma_{R L}=\epsilon_{\mu \nu \rho \lambda \sigma} \operatorname{TrD}_{\mu} \mathrm{hF}_{\mathrm{R}, \nu \rho} \mathrm{h}^{\dagger} \mathrm{F}_{\mathrm{L}, \lambda \sigma} \\
& \Gamma_{L R}=\epsilon_{\mu \nu \rho \lambda \sigma} \operatorname{TrD}_{\mu} \mathrm{hh}^{\dagger} \mathrm{F}_{\mathrm{L}, \nu \rho} \mathrm{hF}_{\mathrm{R}, \lambda \sigma} \mathrm{h}^{\dagger}
\end{aligned}
$$

The covariantly transforming field strengths are

$$
\begin{aligned}
& F_{L, \mu \nu}=\partial_{\mu} A_{L, \nu}-\partial_{\nu} A_{L, \mu}-i\left[A_{L, \mu}, A_{L, \nu}\right] \\
& F_{R, \mu \nu}=\partial_{\mu} A_{R, \nu}-\partial_{\nu} A_{R, \mu}-i\left[A_{R, \mu}, A_{R, \nu}\right] .
\end{aligned}
$$

For the photon, parity invariance fixes $\alpha=1 / 2$. The last four terms contain the process $\pi \rightarrow 2 \gamma$.

I may seem to be drifting from the lattice aspects of the chiral symmetry problem. My purpose in this discussion was to point out how certain field theoretical phenomena require the introduction of extra dimensions. These effects are closely tied to anomalies. The message is that for chiral symmetry on the lattice, anomalies might lead to extra dimensions, just as they have for this simple effective Lagrangian. 


\section{Topology and zero modes}

The mid 70's saw an explosion of interest in topological structures in classical field theories. These were particularly interesting in their non-perturbative properties. On quantization, topological solitons became a new type of particle beyond the simple excitations of the fundamental fields. Structures in four dimensions were found that led to unanticipated tunneling processes, eventually tied to the anomalies alluded to throughout this document. Indeed, usual discussions of the theta parameter start directly with topologically non-trivial classical gauge field configurations. My presentation in terms of chiral Lagrangians relies on using the anomaly to move this parameter into the mass term.

Shortly after the discovery of these topological structures, it was realized that Fermions can behave in rather interesting ways in the presence of non-trivial classical field configurations. One provocative point of view (Jackiw and Rebbi, 1976; Goldstone and Wilczek, 1981) refers to states of "half-integer Fermion number." These modes come about by matching solutions of the Dirac equation in different backgrounds. With appropriate boundary conditions, some solutions only had one state, or chirality, as opposed to the usual doubling due to spin. If the basic topological background defect has spatial extension, such as along a string or membrane, these special states could move. This idea eventually evolved into the basis of the domain-wall formulation. A four dimensional defect in a five dimensional underlying theory can support low energy excitations which mimic free Fermions of a definite chirality.

The connection with anomalies was elegantly presented by Callan and Harvey (1985) who considered a five dimensional space wherein the mass of a Fermion changed sign on a four dimensional interface. Fermionic zero modes appeared on the interface, and were capable of carrying momentum in the transverse direction. At scales well below the basic Fermion mass, the theory reduces to a four dimensional model of massless Fermions. They then discussed how anomalous processes appeared as a flow of eigenmodes into the fifth dimension.

Kaplan (1992) argued that there was nothing in this mechanism that precluded working on a lattice. Indeed, the lattice adds additional insights. The continuum model has an ambiguity about which direction in the fifth dimension the anomalous currents flow. On the lattice, this direction is determined by the sign of the Wilson term, the same quantity that enables the theta parameter to retain its physical significance. Also, once the anomaly is known to go only in a single direction, the interface can be replaced by a surface model, 
simpler for calculations (Shamir, 1993b, 1994).

These surface modes were anticipated considerably earlier in the context of band theory in condensed matter physics. Considering two coupled bands, Shockley (1939) showed that when the coupling between these bands becomes large, the gap between them can close and reopen leaving behind surface states. These states are exactly the surface modes that form the basis of domain-wall Fermions. The one conceptual difference is a particle-antiparticle symmetry, which forces the present states to lie at exactly zero energy. 


\section{The icetray model}

In this section I will approach domain-wall Fermions from a rather unconventional direction. Following a recent paper (Creutz, 1999), I present the subject from a "chemists" point of view, in terms of a chain molecule with special electronic states carrying energies fixed by symmetries. For lattice gauge theory, placing one of these molecules at each space-time site gives excitations of naturally zero mass. This is in direct analogy to the role of chiral symmetry in conventional continuum descriptions.

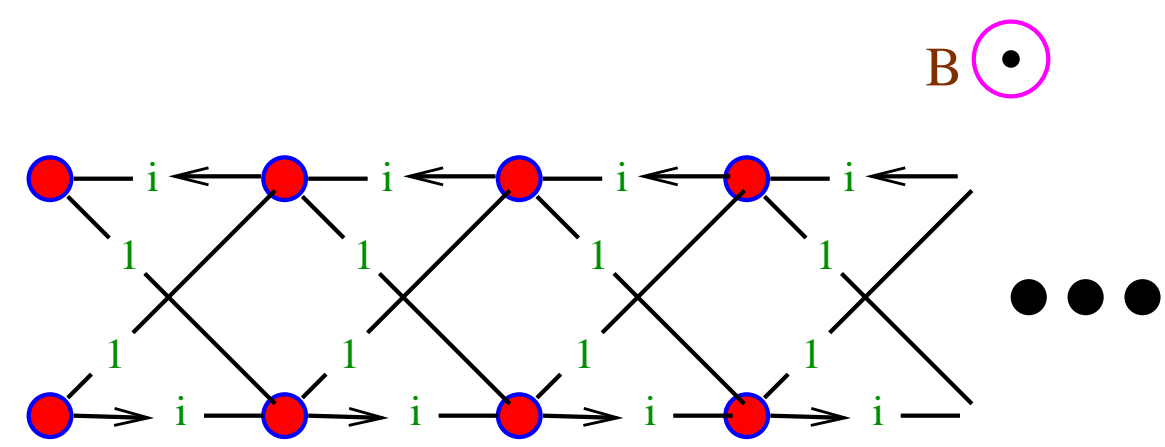

Fig. 15. A ladder molecule in a magnetic field. The phases represent one unit of magnetic flux per plaquette.

To start, consider two rows of atoms connected by horizontal and diagonal bonds, as illustrated in Fig. (15) . The bonds represent hopping terms, wherein an electron moves from one site to another via a creation-annihilation operator pair in the Hamiltonian. Later I will include vertical bonds, but for now consider just the horizontal and diagonal connections.

Years ago during a course on quantum mechanics, I heard Feynman present an amusing description of an electron's behavior when inserted into a lattice. If you place it initially on a single atom, the wave function will gradually spread through the lattice, much like water poured in a single cell of a metal ice cube tray. With damping, it settles into the ground state which has equal amplitude on each atom. To this day I cannot fill an ice cube tray without thinking of this analogy and pouring all the incoming water into a single chamber.

I now complicate this picture by applying a magnetic field orthogonal to the plane of the system. This introduces phases as the electron hops, causing interesting interference effects. In particular, consider a field of one-half flux unit per plaquette. This means that when a particle hops around a unit area (in terms of the basic lattice spacing) the 


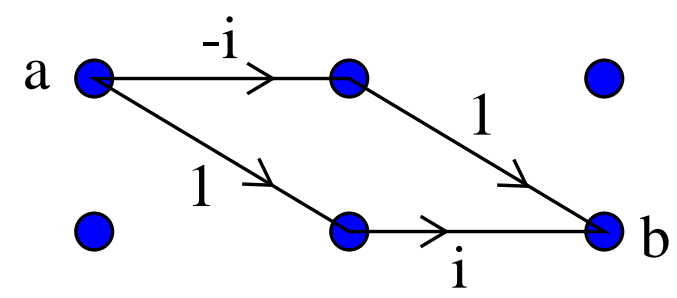

Fig. 16. The two paths from site $\mathbf{a}$ to site $\mathbf{b}$ cancel quantum mechanically when one half flux unit passes through the loop.

wave function picks up a minus sign. Just where the phases appear is a gauge dependent convention; only the total phase around a closed loop is physical. One choice for these phases is indicated by the numbers on the bonds in Fig. (15). The phase factors cause cancellations and slow diffusion. For example, consider the two shortest paths between the sites $\mathbf{a}$ and $\mathbf{b}$ in Fig. (16) . With the chosen flux, these paths exactly cancel. For the full molecule this cancellation extends to all paths between these sites. An electron placed on site $\mathbf{a}$ can never diffuse to site $\mathbf{b}$. Unlike in the ice tray analogy, the wave function will not spread to any site beyond the five nearest neighbors.

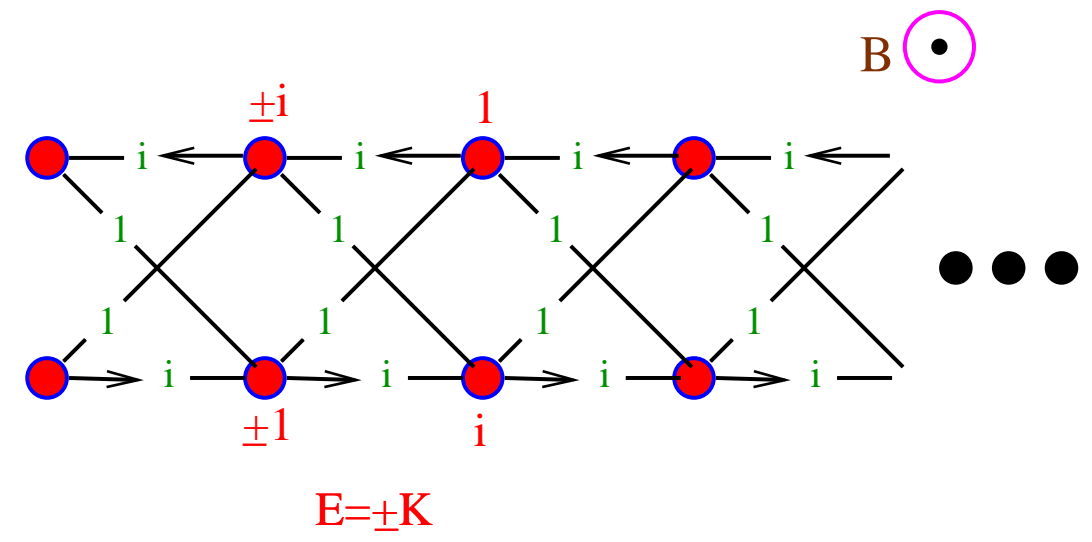

Fig. 17. There exist two eigenstates per plaquette that do not spread with time.

The states have opposite signs for the energy.

As a consequence, the Hamiltonian has localized eigenstates. There are two such states per plaquette; one possible representation for these two states is shown in Fig. (17) . The states are restricted to the four sites labeled by their relative wave functions. Their energies are fixed by the size of the hopping parameter $K$.

For a finite chain of length $L$ there are $2 L$ atoms, and thus there should be a total of $2 L$ possible states for the electron (ignoring spin for the moment). There are $L-1$ plaquettes, and thus $2 L-2$ of the above localized states. This is almost the entire spectrum of the 


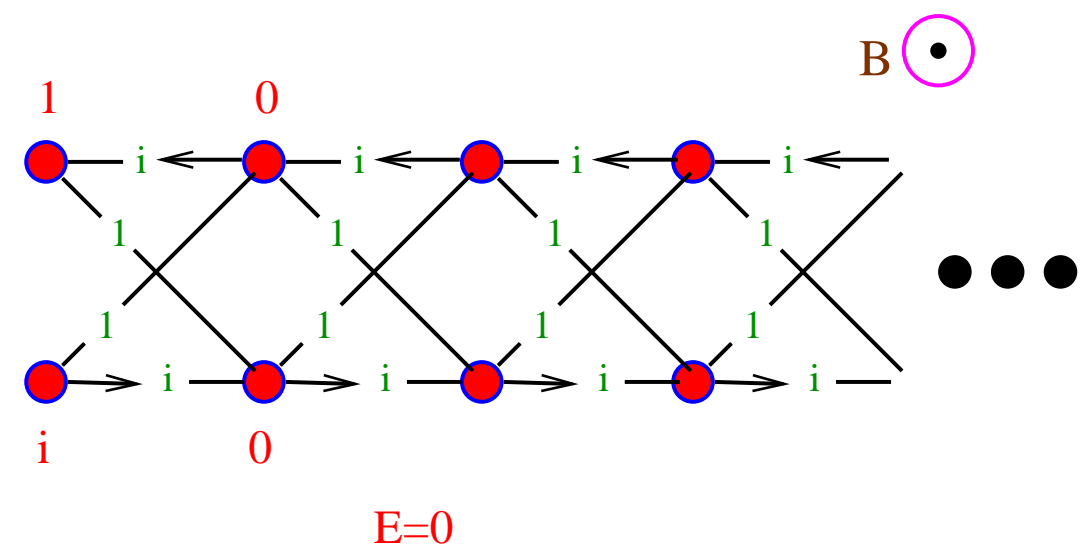

Fig. 18. One eigenstate of exactly zero energy is bound on the end of the chain.

Hamiltonian, but two states are left over. These are zero energy states bound to the ends of the system. The wave function for one of those is shown in Fig. (18) . This is the full spectrum of the Hamiltonian: $L-1$ degenerate states of positive energy, a similar number of degenerate negative energy states, and two states of zero energy bound on the ends.

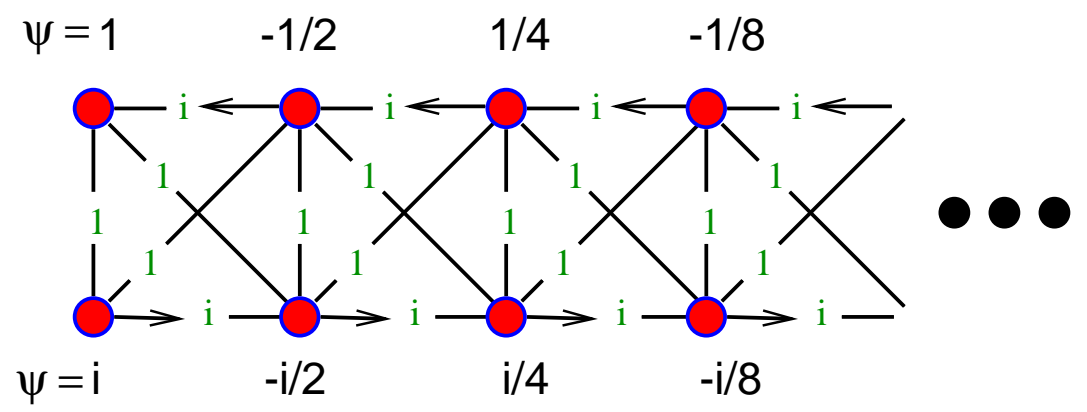

Fig. 19. The zero energy state is robust under the addition of vertical bonds, although the wave function now has an exponential decrease into the molecule.

Now consider what happens when vertical bonds are included in this molecule. The phase cancellations are no longer complete and the localized states spread to form two bands, one with positive and one with negative energy. However, for the present purposes, the remarkable result is that the zero modes bound on the ends of the chain are robust. The corresponding wave functions are no longer exactly located on the last atomic pair, but have an exponentially suppressed penetration into the chain. Fig. (19) shows the wave function for one of these states when the vertical bond has the same strength as the others. There is a corresponding state on the other end of the molecule.

When the chain is very long, both of the end states are forced to zero energy by symmetry considerations. First, since nothing distinguishes one end of the chain from 
the other, they must have equal energy, $E_{L}=E_{R}$. On the other hand, a change in phase conventions, effectively a gauge change, can change the sign of all the vertical and diagonal bonds. Following this with a left-right flip of the molecule will change the signs of the horizontal bonds. This takes the Hamiltonian to its negative, and shows that the states must have opposite energies, $E_{L}=-E_{R}$. This is also a consequence of a particlehole symmetry. The combination of these results forces the end states to zero energy, with no fine tuning of parameters.

For a finite chain, the exponentially decreasing penetration of the end states into the molecule induces a small interaction between them. They mix slightly to acquire exponentially small energies $E \sim \pm e^{-\alpha L}$. As the strength of the vertical bonds increases, so does the penetration of the end states. At a critical strength, the mixing becomes sufficient that the zero modes blend into the positive and negative energy bands. In the full model, the mixing depends on the physical momentum, and, as I will discuss shortly, this disappearance of the zero modes is the mechanism that removes the "doublers" when spatial momentum components are near $\pi$ in lattice units (Jansen and Schmaltz, 1992; Creutz and Horvath, 1994). 


\section{Domain-wall Fermions}

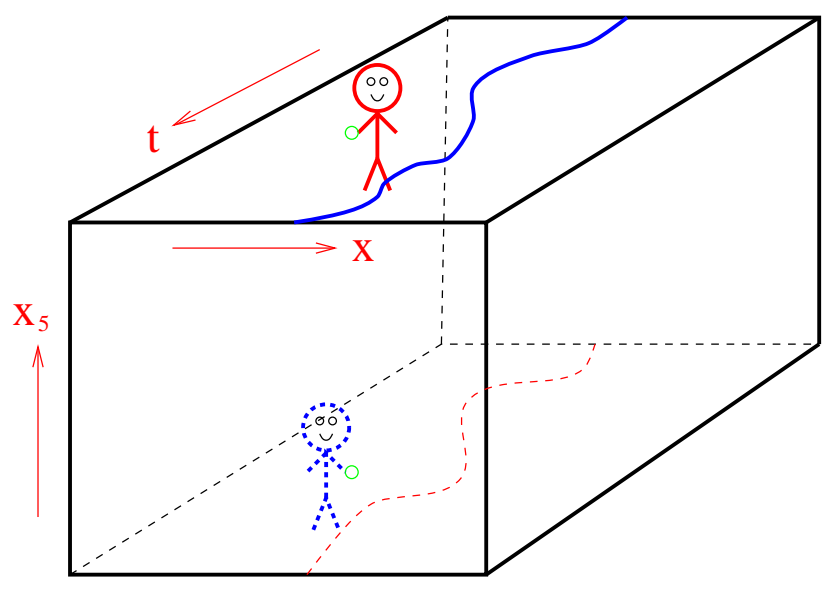

Fig. 20. The basic domain-wall formalism has the physical particles bound on the surfaces of a five dimensional world. Left and right handed partners lie on opposing walls.

These energy levels forced to zero by symmetry lie at the core of the domain wall idea. On every spatial site of a three dimensional lattice place one of these chain molecules. The distance along the chain is usually referred to as a fictitious "fifth" dimension. The different spatial sites are coupled, allowing particles in the zero modes to move around. These are the physical Fermions. The symmetries that protect the zero modes now protect the masses of these particles. Their masses receive no additive renormalization, exactly the consequence of chiral symmetry in the continuum. The physical picture is sketched in the cartoon of Fig. (20) . For this figure I have rotated the fifth dimension to the vertical. The world lines of everyday objects traverse the four dimensional surface of this five dimensional manifold. The model is mathematically identical to five dimensional Wilson Fermions at supercritical hopping.

Actually, the connection with chiral symmetry is much deeper than just an analogy. The construction guarantees that the modes are are automatically chiral. To see how this works, first note that the construction of the hopping in the fifth dimension is consistent 
with a particular representation of the Dirac gamma matrices

$$
\begin{aligned}
\gamma_{0} & =\left(\begin{array}{ll}
0 & 1 \\
1 & 0
\end{array}\right) \\
\gamma_{5} & =\left(\begin{array}{cc}
0 & -i \\
i & 0
\end{array}\right) \\
\gamma_{i} & =\left(\begin{array}{cc}
\sigma_{i} & 0 \\
0 & -\sigma_{i}
\end{array}\right) .
\end{aligned}
$$

Note how Pauli spin matrices enter the spatial hoppings. To see their effect, it is easiest to go to momentum space in the four physical dimensions. Then the spatial hoppings become diagonal and generate effective vertical bonds which depend on momentum. Including a diagonal term proportional to a parameter $M$ as before, the net vertical bonds are parametrized by the matrix

$$
\left(\begin{array}{cc}
0 & M+K_{4}\left(\cos \left(q_{i}\right)+i \sigma_{i} \sin \left(q_{i}\right)\right) \\
M+K_{4}\left(\cos \left(q_{i}\right)-i \sigma_{i} \sin \left(q_{i}\right)\right) & 0
\end{array}\right) .
$$

I have explicitly kept the hopping parameter $K_{4}$ and distinguished it with the subscript 4 to denote the four physical dimensions. I will discuss shortly reasons for distinguishing the hopping in the physical from the hopping in the fifth direction.

Here the terms involving $\sigma \sin (q)$ move the surface modes from zero energy to a finite value $E^{2} \sim K_{4}^{2} \sum \sin ^{2}\left(q_{i}\right)$. The group velocity contains a factor of the Pauli matrices, indicating that the low energy and low momentum modes are chiral. Note that at small momentum this is exactly the naive lattice Fermion spectrum. Were that to continue to larger momentum, there would be doublers when any component of the momentum is near $\pi$. Removing them involves the terms involving $\cos (q)$, which come from the Wilson term in the action. As discussed before, the surface modes are robust under the addition of a real vertical bond, and thus to first approximation the $M$ and $\cos (q)$ terms in Eq. (69) are unimportant. However, such terms do influence the penetration of the modes into the extra dimension, and if they become too large, then these modes can disappear. This is useful, since the Wilson terms can make the surface modes disappear before the momentum reaches the doubling points. In other words, $M$ and $K_{4}$ should take values such that the surface modes are present at small $q$, but must also be in a region so that the modes are lost before reaching the doubler points.

So far I have been treating only free Fermions. To couple gauge fields without adding lots of unneeded degrees of freedom, it is simplest to only have gauge fields in the physical 
directions. In this approach, the extra dimension is perhaps best thought of as a flavor space (Narayanan and Neuberger, 1993a,b, 1994, 1995). With a finite lattice this procedure gives equal couplings of the gauge field to the Fermion modes on opposing walls in the extra dimension. The result is an effective light Dirac Fermion. In the case of the strong interactions, this provides an elegant scheme for a natural chiral symmetry without the tuning inherent in the usual Wilson approach. The breaking of chiral symmetry arises only through finiteness of the extra dimension.

In usual discussions of domain-wall Fermions, no distinction is made between the strength of the hopping in the spatial dimension and that in the extra direction. For free Fermions this works well, but Brower and Svetitsky (2000) have argued that in the strong coupling limit the doublers can cause difficulties. While their discussion was in Hamiltonian language, it is likely to carry through for the Lagrangian discussion as well. To resolve this, note that the structure of the free theory is quite rich if the strength of the spatial hopping, $K_{4}$ and that in the extra dimension $K_{5}$ are varied separately. In Fig. 21. I sketch the number of independent Fermion species present in the $\left(K_{4}, K_{5}\right)$ plane for fixed $M=1$. The lines here are places where massless Fermions appear via a vanishing mass gap for the full five dimensional theory. When gauge fields are turned on, all of these lines are expected to be renormalized. The Brower and Svetitsky result suggests that these lines shift to larger $K_{4}$, pinching out the desired phase with only one zero mode on the surface. Whether this pinching is complete at finite gauge coupling or only in the strong coupling limit is at present unresolved.

When the size of the extra dimension is finite, there is a small mixing of the modes on the two walls. Regarded as a theory of light Fermions plus a set of heavier fields associated with the fifth dimension, this mixing generates a small residual mass for the low energy states. For the strong interactions one wants to include quark masses anyway, so for practical calculations one might as well work with finite $L_{5}$, including this mixing as part of the physical quark masses. This point of view is controversial - integrating out the heavy fields yields a rather complicated non-local effective theory, making the interpretation of this mixing as a mass less clear (Kikukawa, 1999). Nevertheless, this residual mixing does play a crucial role towards the physics discussed extensively in the sections on the phase of quark masses. The angle $\Theta$ discussed there is effectively the relative phase a quark acquires in tunneling through the extra dimension compared to any extrinsic mass added to the theory. I will return to this point later. 


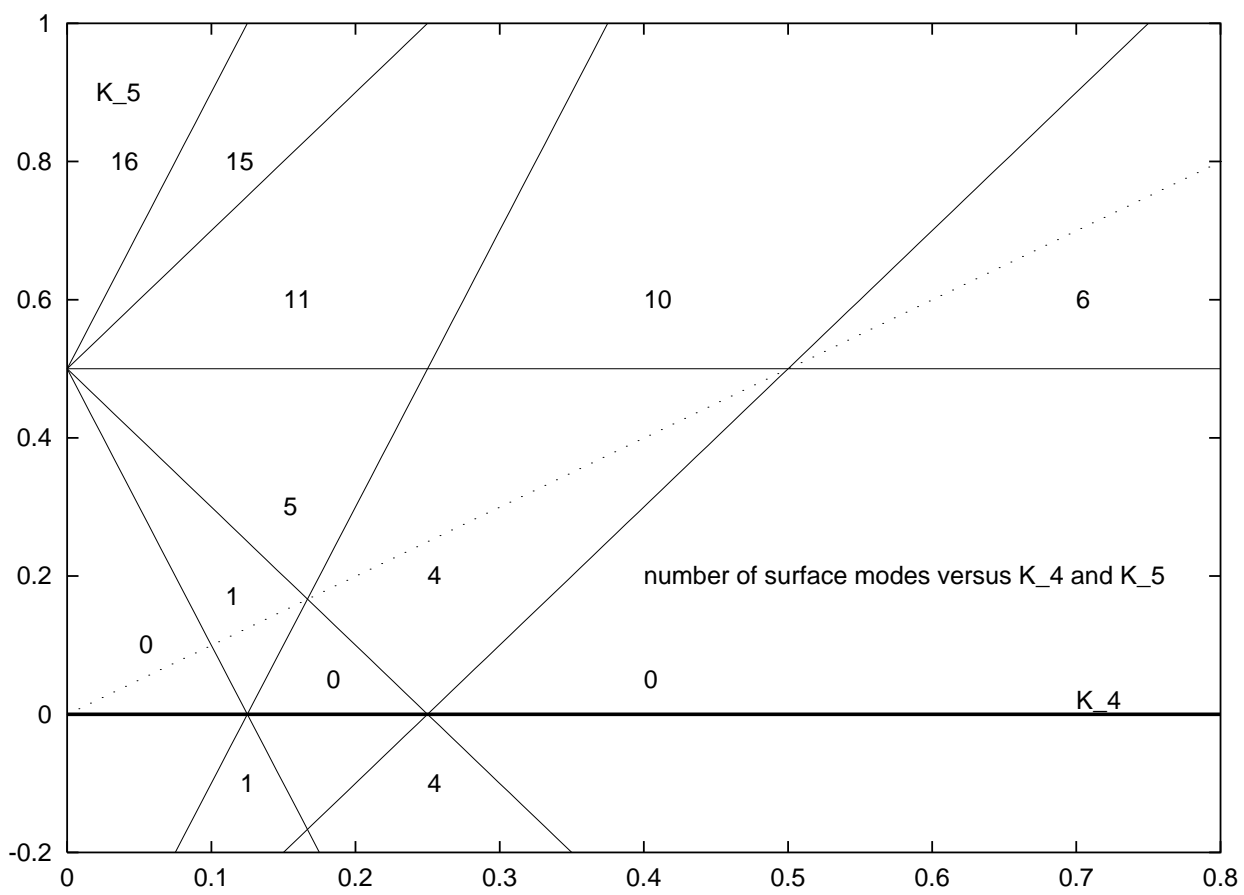

Fig. 22. A rich phase structure appears when the four dimensional hopping parameter $K_{4}$ is allowed to vary separately from $K_{5}$ corresponding to the extra dimension. The lines indicate the loci of massless five dimensional Fermions. The numbers labeling the various regions are the number of independent massless modes propagating on the $4 \mathrm{~d}$ surface of a five dimensional lattice. Usual domainwall Fermions utilize the region with only one surface mode.

In the domain-wall scheme there are a large number of heavy fields. These can cause difficulties for perturbative studies. The usual way to eliminate this problem is to add in a comparable number of auxiliary Bosonic fields (Frolov and Slavnov, 1994; Narayanan and Neuberger, 1993a; Furman and Shamir, 1995). These use the same action as the Fermions, but with periodic rather than open boundary conditions. In loops they will cancel the heavy Fermions, eliminating the need for large perturbative counter-terms. This is a useful technical point, but not essential to the basic understanding of the formulation.

One issue with domain-wall Fermions is that the two helicities on opposite walls are both present, so this is not a chiral gauge theory of the type needed to describe the weak interactions. The main unresolved question is whether there is some scheme to make the opposite helicity states on one wall either decouple or move to large mass. I return to this topic in later sections. Before that, however, I digress and discuss a physical picture of how anomalies work in this five dimensional model. 


\section{Anomalies in the domain-wall approach}

These surface modes form an elegant formulation of a $d$ dimensional Fermion theory in terms of surface modes in a $d+1$ dimensional underlying space. However, it is a bit mysterious how anomalies can work when the left and right zero modes are so strongly separated. In this section I follow the discussion of Creutz and Horvath (1994), showing how anomalous processes appear as a flow of states in a Hamiltonian formulation. The approach is motivated by the earlier discussion of the anomaly, and displays a mechanism to absorb the rising eigenvalues without having an infinite band.

The basic picture returns to the continuum context of Callan and Harvey (1985). A vector theory with a mass term having a domain-wall shape in an extra dimension has chiral zero-modes living on the wall. Anomalous currents associated with this zero-mode must be absorbed in the underlying $d+1$ dimensional theory since that world is anomaly free. What on the interface looks like an anomaly is the flow of charge into the extra dimension and the role of the heavy modes is to carry that charge. Since opposite chirality partners live on opposite walls, the charge has to be transported through the extra dimension.

For simplicity I concentrate on the one dimensional case with gauge group $U(1)$. An external $U(1)$ gauge field is manifested in phase factors appearing whenever a Fermion hops from one spatial site to the next. Calling this factor $U(k)=e^{i \alpha_{k}}$ for the hopping from space site $k$ to $k+1$, the Wilson Hamiltonian is

$$
\begin{aligned}
H=\sum_{k, j} & \left(K \bar{\psi}_{k, j+1}\left(\gamma_{5}-1\right) \psi_{k, j}-K \bar{\psi}_{k, j}\left(\gamma_{5}+1\right) \psi_{k, j+1}\right. \\
& +K \bar{\psi}_{k+1, j} U(k)\left(\gamma_{1}-1\right) \psi_{k, j}-K \bar{\psi}_{k, j} U^{\dagger}(k)\left(\gamma_{1}+1\right) \psi_{k+1, j} \\
& \left.+M \bar{\psi}_{k, j} \psi_{k, j}\right) .
\end{aligned}
$$

Here $k$ represents the spatial coordinate and $j$ the "fifth" dimension. I have taken the Wilson parameter $r=1$ and hopping parameter $K=K_{4}=K_{5}$. I take antiperiodic boundary spatial conditions $\psi_{L, j}=-\psi_{0, j}$ for the Fermion field. Of course, for domainwall Fermions the boundaries in the extra dimension are open.

A gauge transformation by a phase $g$ at site $k$ takes $U(k)$ to $U(k) g^{\dagger}, U(k+1)$ to

$g U(k+1)$, and $\psi_{k}$ to $g \psi_{k}$. The invariance of the Hamiltonian under this symmetry implies that the spectrum only depends on the product of all the $U(k)$ around the periodic spatial lattice. This is the net phase acquired by a Fermion traveling all the way around the finite periodic system. 
A convenient gauge choice is to evenly distribute the phases so that $U(k)=e^{i \alpha}$ is independent of $k$. This keeps momentum space simple, with the Hamiltonian becoming

$$
\begin{aligned}
H=\sum_{q, j} & \left(K \bar{\psi}_{q, j+1}\left(\gamma_{5}-1\right) \psi_{q, j}-K \bar{\psi}_{q, j}\left(\gamma_{5}+1\right) \psi_{q, j+1}\right. \\
& +2 i K \sin (2 \pi q / L-\alpha) \bar{\psi}_{q, j} \gamma_{1} \psi_{q, j} \\
& \left.+(M-2 K r \cos (2 \pi q / L-\alpha)) \bar{\psi}_{q, j} \psi_{q, j}\right) .
\end{aligned}
$$

Here $j$ labels the fifth coordinate and the spatial momentum variable $q$ runs from 0 to L. The energy eigenstates are functions of the momentum shifted by $\alpha$. As $\alpha$ increases from 0 to $2 \pi / L$, sequential momenta rotate into each other. The total net phase in this case is $2 \pi$ and, as expected, physics goes back to itself. In natural units, one unit of flux represents $\alpha=2 \pi / L$.

In the adiabatic limit, time evolution is a continuous flow of one particle states with $\alpha$. For the various eigenstates of the Hamiltonian on a small system, Creutz and Horvath (1994) calculated the expectation value of the fifth coordinate. Fig. (23), taken from that paper, shows the results for four distinct values of $\alpha=0, \frac{1}{4}, \frac{1}{2}, \frac{3}{4}$. States with a low magnitude for the energy lie at the lattice ends and rise or fall with $\alpha$ without substantially changing their position in the extra dimension. The same is true for the very high energy states, residing deep in the lattice interior. As their energy increases, the surface states penetrate further into the extra dimension. When the energy of such a level gets close to the cutoff, it moves swiftly towards the middle. At the same time, another level from the middle lowers its energy and runs towards the opposite wall. This is also true for corresponding levels with negative energy; they just move in the opposite direction. The heavy modes near the cutoff are responsible for carrying the charge on and off the surfaces.

On the lattice there is considerable freedom to define an axial charge; any definition assigning opposite charges to the two zero-modes living on the opposite walls should yield a correct continuum limit. One possibility is to define the axial charge as the Fermion number weighted by the location in the extra dimension

$$
Q_{5}=\frac{1}{L_{5}-1} \sum_{q, j}\left(L_{5}-1-2 j\right) \psi_{q, j}^{\dagger} \psi_{q, j}
$$

with $j=0,1,2, \ldots, L_{5}-1$. This assigns to a one particle state +1 if it is exactly bound to the left wall with $j=0$ and -1 if it is bound to the right wall. For states smeared 


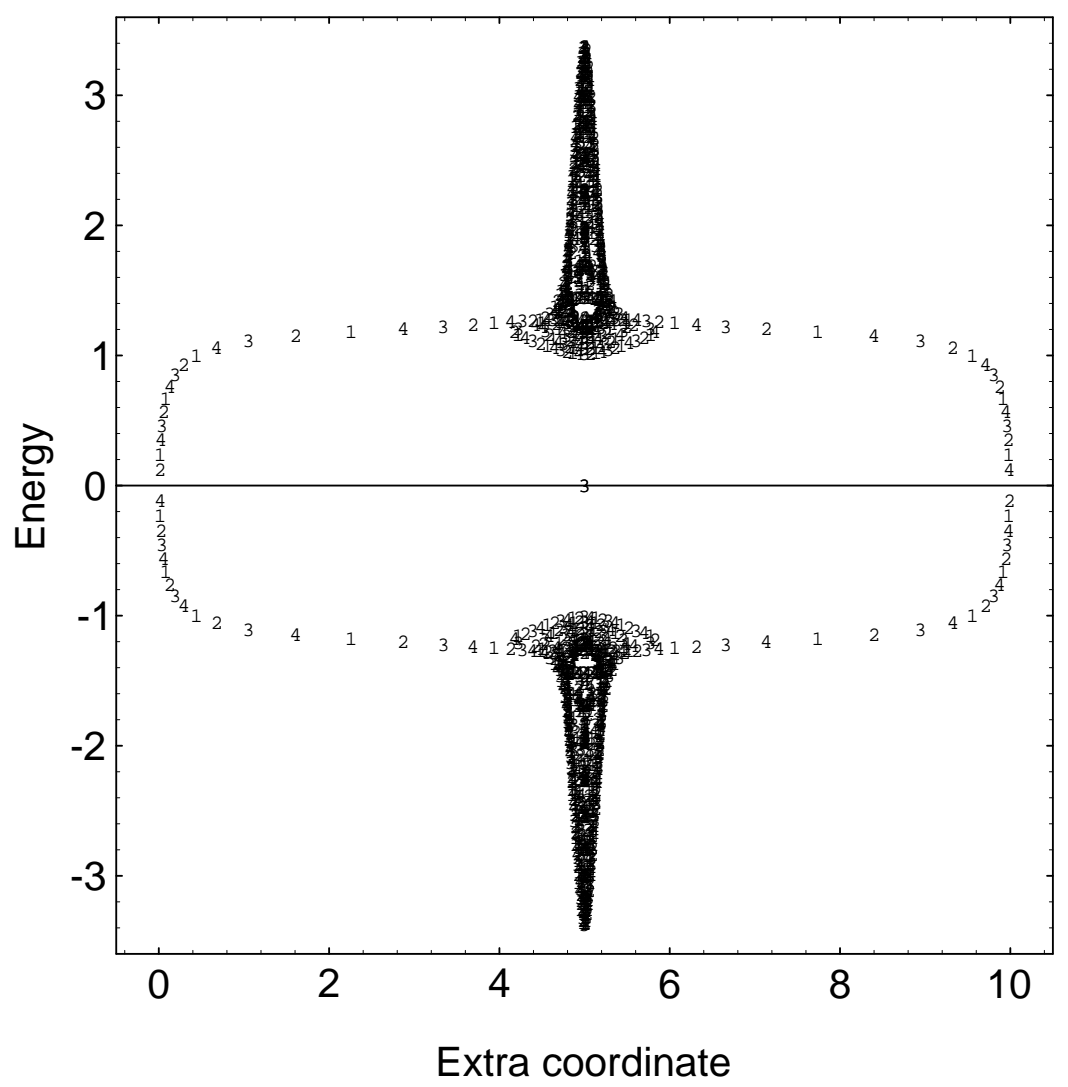

Fig. 23. The energy spectrum as a function of $\left\langle x_{5}>\right.$ for $\alpha=0, \frac{1}{4}, \frac{1}{2}, \frac{3}{4}$ on a $L_{5}=11$ by $L=16$ lattice. Successive positions of energy levels are marked by $1,2,3$ and 4 respectively. The levels rotate in an anti-clockwise sense.

uniformly over $j$, we obtain zero. Regarding the extra dimension as an internal space, we see that the axial charge is nothing but a particular combination of flavor charges.

The vacuum of the theory fills all the negative energy eigenstates. Physical energies are taken relative to this state. At zero $\alpha$, the vacuum has zero axial charge because of the mirror symmetry of the Hamiltonian with respect to the middle of the extra space $\left(j \rightarrow L_{5}-1-j\right)$. To see the anomaly, evolve the vacuum in an adiabatic field, increasing the value of $\alpha$ from 0 to 1 unit of flux and look for the change in the total axial charge. As the field is turned on, the levels in the Dirac sea start to move anti-clockwise $(\alpha>0)$, increasing the total axial charge.

When the value of the field is close to one half unit of flux, one of the filled levels on 
the right surface is just about to become the positive energy particle and one empty level on the left is just about to drop into the sea. However, as long as the extra dimension is finite, the surface states are not exactly massless. There is always a tiny mass $\delta$ present, caused by mixing of the states on the opposite walls. When the fields are truly adiabatic, with the typical time $\tau$ for turning on the fields longer than any other time scale in the problem, e.g. $\tau \gg 1 / \delta$, instead of creation of a particle-hole pair, the two levels will have enough time to exchange between the walls. As a consequence, there is a jump at $\alpha=1 / 2$, which allows vacuum to evolve into its original state as $\alpha$ approaches one unit of flux. This is the reason for the appearance of the point labeled with " 3 " in the exact center of Fig. (23) . 


\section{Weak interactions and mirror Fermions}

With an exact gauge invariance and a finite size for the extra dimension, the surface models are inherently vector-like. The Fermions always appear with both chiralities, albeit separated in the extra dimension. However, it is an experimental fact that only left handed neutrinos couple to the weak Bosons. In this section I discuss one way (Creutz and Horvath, 1994) to break the symmetries between these states, resulting in a theory with only one light gauged chiral state. Here I keep the underlying gauge symmetry exact, but do require that the chiral gauge symmetry be spontaneously broken, just as observed in the standard model. The picture also contains heavy mirror Fermions. If anomalies are not canceled amongst the light species, these heavy states must survive in the continuum limit. It remains an open question when anomalies are properly canceled whether it might be possible to drive the heavy mirror states to arbitrarily large mass.

I start with two separate species $\psi_{1}$ and $\psi_{2}$ in the surface mode picture. However, I treat these in an unsymmetric way. For $\psi_{1}$ use the previous Hamiltonian. For $\psi_{2}$ change the sign of all terms proportional to $\gamma_{5}$. On a given wall, the surface modes associated with $\psi_{1}$ and $\psi_{2}$ will then have opposite chirality.

Now introduce the gauge fields. Since I want to eventually couple only one-handed neutrinos to the vector Bosons, consider gauging $\psi_{1}$ but not $\psi_{2}$. Indeed, at this stage $\psi_{2}$ represents a totally decoupled right handed "spectator" Fermion on one wall. A mirror situation appears on the opposite wall, consisting of a right handed gauged state and a left handed decoupled Fermion.

The next ingredient is to spontaneously break the gauge symmetry, as in the standard model, by introducing a Higgs field $\phi$ with a non-vanishing expectation value. This field can generate masses as in the standard model by coupling $\psi_{1}$ and $\psi_{2}$ with a term of the form $\bar{\psi}_{1} \psi_{2} \phi$.

The new feature is to allow the coupling to the Higgs field to depend on the extra coordinate. In particular, let it be small or vanishing on one wall and large on the other. The surface modes are then light on only the first wall.

Fig. (24), taken from Creutz and Horvath (1994), considers one space dimension and sketches the Fermion spectrum of this model with vanishing gauge fields and a constant Higgs field. As in other mirror Fermion models (Montvay, 1987, 1993), triviality arguments suggest bounds on the masses of the heavy particles. This is certainly the case when the light Fermions alone give an anomalous gauge theory, in which case the mirror particles 


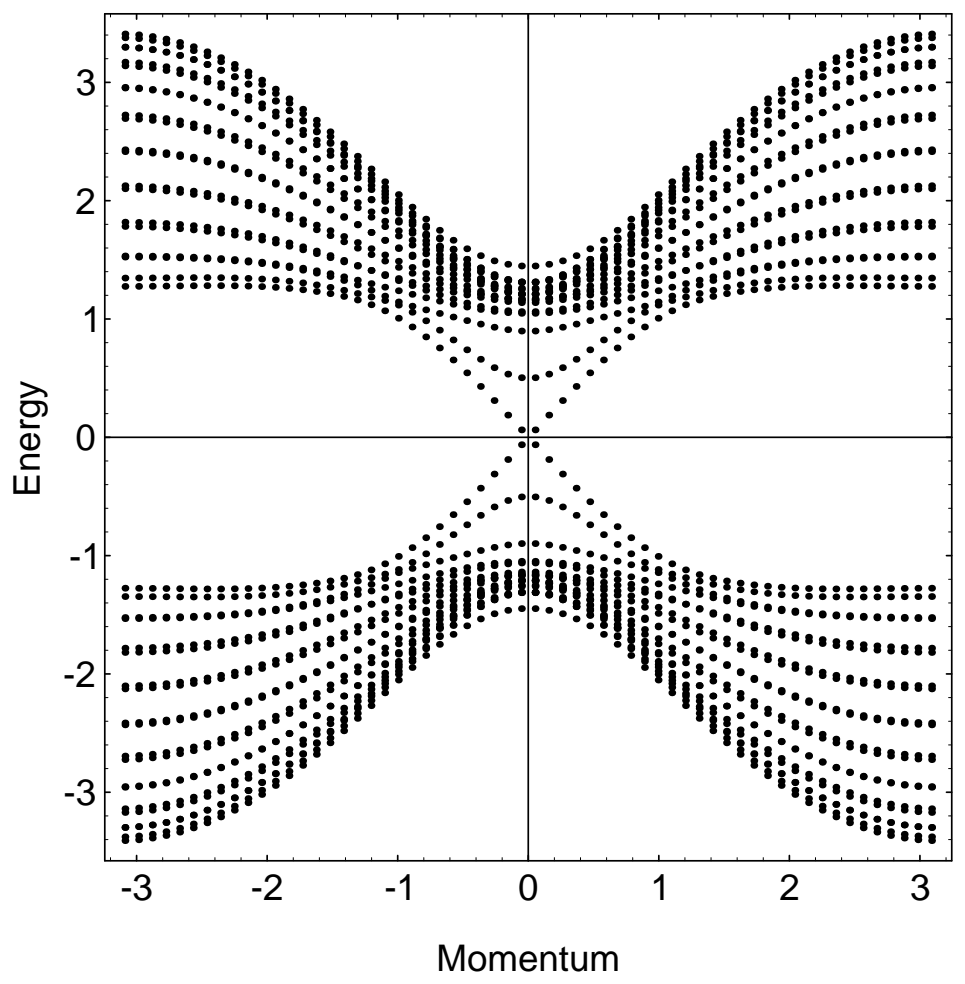

Fig. 24. Energy levels versus momentum for the two species model discussed in the text. Here the lattice is $L_{5}=10$ by $L=60$, and the Higgs coupling is switched off at the middle of the extra dimension. Note how one species is effectively massless and the other massive.

cannot become much heavier than the vector mesons, i.e. the $W$. Golterman and Shamir (1985) have shown that naively taking the Higgs-Fermion coupling to infinity on one wall can introduce a plethora of new low energy bound states. It is conceivable that the restrictions on the mirror Fermion masses are weaker when anomalies cancel amongst the light states. In this case there is no perturbative need for the heavy states, and perhaps they can be driven to infinite mass in the continuum limit. If so, this would be a candidate for a lattice discretization of the standard model.

This model, however, does not lead to baryon number violation (Distler and Rey, 1993). The anomaly will involve a tunneling of baryons from one wall to the opposite, where they become mirror baryons. Even if these extra particles are heavy, the decay can only occur through mixing with the ordinary particle states. In this sense, the mirror 
particles still show their presence in low energy physics.

A speculative proposal is to use the right handed mirror states in some way as observed particles. Indeed, the world has left handed leptons and right handed anti-baryons. Any simple extension of this idea to a realistic model must unify these particles. The next section presents a possible construction along this line. 


\section{Leptons and anti-quarks}

Elementary particle interactions are parity violating, i.e. the theory must be chiral. This is implemented in the standard model of elementary particle interactions via the coupling of the electroweak bosons to chiral currents. How to formulate such a theory on the lattice remains controversial. Some time ago Eichten and Preskill (1986) pointed out that a lattice approach must accomodate the 't Hooft (1976a,b) baryon decay process. Thus any fully finite theory must incorporate baryon violating terms in the underlying Lagrangian. Despite difficulties (Golterman, Petcher, and Rivas, 1993) with their specific model, the idea of including such terms remains compelling. In this section I will discuss a specific recent scheme (Creutz, Tytgat, Rebbi, and Xue, 1997) building on the domain-wall approach. This approach has not been widely accepted due to a rather complex action. Nevertheless, I hope the following interpretation of the standard model will at least seem provocative.

The standard model is based on the product of three gauge groups, $S U(3) \times S U(2) \times$ $U(1)_{e m}$. Here the $S U(3)$ represents the strong interactions of quarks and gluons, the $U(1)_{e m}$ corresponds to electromagnetism, and the $S U(2)$ gives rise to the weak interactions. I gloss over the technical details of electroweak symmetry breaking and the mixing between the $U(1)$ field and the neutral $S U(2)$ gauge Boson.

The full model is, of course, parity violating, as necessary to describe observed helicities in beta decay. This violation is normally considered to lie in the $S U(2)$ of the weak interactions, with both the $S U(3)$ and $U(1)_{e m}$ being parity conserving. However, this is actually a convention, adopted primarily because the weak interactions are small. I argue below that reassigning degrees of freedom allows a reinterpretation where the $S U(2)$ gauge interaction is vector-like. Since the full model is parity violating, this process shifts the parity violation into the strong, electromagnetic, and Higgs interactions. The resulting theory pairs the left handed electron with a right handed anti-quark to form a Dirac Fermion.

With a vector-like weak interaction, the chiral issues which complicate lattice formulations now move to the other gauge groups. Requiring gauge invariance for the re-expressed electromagnetism then clarifies the mechanism behind a recent proposal for a lattice regularization of the standard model.

To begin, consider only the first generation, which involves four left handed doublets. These correspond to the neutrino/electron lepton pair plus three colors for the up/down 
quarks

$$
\left(\begin{array}{c}
\nu \\
e^{-}
\end{array}\right)_{L},\left(\begin{array}{l}
u^{r} \\
d^{r}
\end{array}\right)_{L},\left(\begin{array}{l}
u^{g} \\
d^{g}
\end{array}\right)_{L},\left(\begin{array}{l}
u^{b} \\
d^{b}
\end{array}\right)_{L} .
$$

Here the superscripts from the set $\{r, g, b\}$ represent the internal $S U(3)$ index of the strong interactions, and the subscript $L$ indicates left-handed helicities.

If I ignore the strong and electromagnetic interactions, leaving only the weak $S U(2)$, each of these four doublets is equivalent and independent. I now arbitrarily pick two of them and do a charge conjugation operation, thus switching to their antiparticles

$$
\begin{aligned}
& \left(\begin{array}{l}
u^{g} \\
d^{g}
\end{array}\right)_{L} \longrightarrow\left(\frac{\overline{d^{g}}}{u^{g}}\right)_{R} \\
& \left(\begin{array}{c}
u^{b} \\
d^{b}
\end{array}\right)_{L} \longrightarrow\left(\frac{\overline{d^{b}}}{u^{b}}\right)_{R} .
\end{aligned}
$$

In four dimensions anti-Fermions have the opposite helicity; so, I label these new doublets with $R$ representing right-handedness.

With two left and two right handed doublets, I can combine them into Dirac fields (Lee and Schrock, 1988)

$$
\left(\begin{array}{l}
\left(\begin{array}{c}
e^{-} \\
\bar{d}^{g}
\end{array}\right)_{L} \\
\left(\frac{d^{g}}{u^{g}}\right)_{R}
\end{array}\right) \quad\left(\begin{array}{l}
\left(\begin{array}{c}
u^{r} \\
d^{r}
\end{array}\right)_{L} \\
\left(\overline{\frac{d^{b}}{u^{b}}}\right)_{R} .
\end{array}\right)
$$

Formally in terms of the underlying fields, the construction takes

$$
\begin{aligned}
\psi & =\frac{1}{2}\left(1-\gamma_{5}\right) \psi_{\left(\nu, e^{-}\right)}+\frac{1}{2}\left(1+\gamma_{5}\right) \psi_{\left(\overline{d^{g}}, \overline{u^{g}}\right)} \\
\chi & =\frac{1}{2}\left(1-\gamma_{5}\right) \psi_{\left(u^{r}, d^{r}\right)}+\frac{1}{2}\left(1+\gamma_{5}\right) \psi_{\left(\overline{d^{b}}, \overline{u^{b}}\right)} .
\end{aligned}
$$

From the conventional point of view these fields have rather peculiar quantum numbers. For example, the left and right parts have different electric charges. Electromagnetism now violates parity. The left and right parts also have different strong quantum numbers; the strong interactions violate parity as well. Finally, the components have different masses; parity is violated in the Higgs mechanism.

The different helicities of these fields also have variant baryon number. This is directly related to the known baryon violating processes through weak "instantons" and axial anomalies ('t Hooft, 1976a,b). As discussed earlier, when a topologically non-trivial weak field is present, the axial anomaly arises from a level flow out of the Dirac sea (Ambjorn, Greensite, and Peterson 1983; Holstein, 1993). This generates a spin flip in the fields, i.e. 
$e_{L}^{-} \rightarrow\left(\overline{u^{g}}\right)_{R}$. Because of my peculiar particle identification, this process does not conserve charge, with $\Delta Q=-\frac{2}{3}+1=\frac{1}{3}$. This would be a disaster for electromagnetism were it not for the fact that simultaneously the other Dirac doublet also flips, $d^{r}{ }_{L} \rightarrow\left(\overline{u^{b}}\right)_{R}$, with a compensating $\Delta Q=-\frac{1}{3}$. This is anomaly cancellation, with the total $\Delta Q=\frac{1}{3}-\frac{1}{3}=0$. Only when both doublets are considered together is the $U(1)$ symmetry restored. In this process baryon number is violated, with $L+Q \rightarrow \bar{Q}+\bar{Q}$. This is the famous " tt Hooft vertex."

This discussion has been in continuum language. Now I return to the lattice, and use the Kaplan-Shamir domain-wall approach. To repeat, in this picture, the four dimensional world is a "4-brane" embedded in 5-dimensions. The complete lattice is a five dimensional box with open boundaries, and the parameters are chosen so the physical quarks and leptons appear as surface zero modes. The elegance of this scheme lies in the natural chirality of these modes as the size of the extra dimension grows. With a finite fifth dimension a doubling phenomenon remains, coming from interfaces appearing as surface/anti-surface pairs. It is natural to couple a four dimensional gauge field equally to both surfaces, giving rise to a vector-like theory.

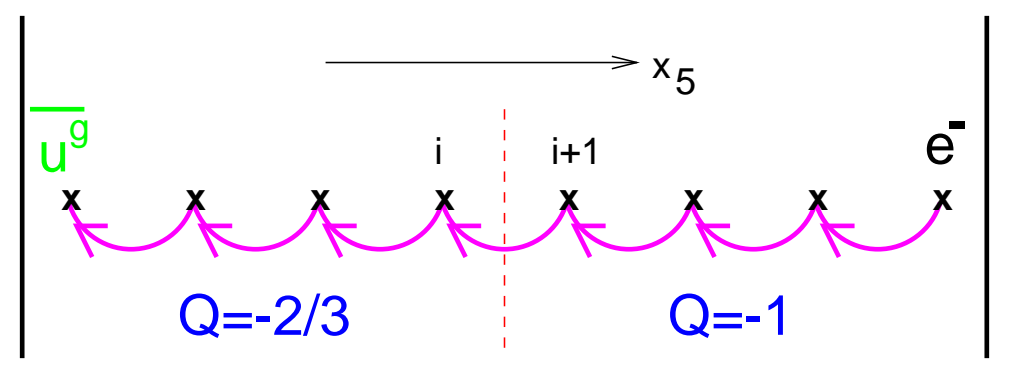

Fig. 25. Pairing the electron with the anti-green-up-quark.

I now insert the above pairing into this five dimensional scheme. In particular, I consider the left handed electron as a zero mode on one wall and the right handed antigreen-up-quark as the partner zero mode on the other wall, as sketched in Fig. (25) . This provides a complete lattice regularization for the $S U(2)$ of the weak interactions.

However, since these two particles have different electric charge, $U(1)_{E M}$ must be broken in the interior of the extra dimension. I now proceed in analogy to the "waveguide" picture (Golterman, Jansen, Petcher, and Vink, 1994) and restrict this charge violation to $\Delta Q$ to one layer at some interior $x_{5}=i$. Then the Fermion hopping term from $x_{5}=i$ to $i+1$

$$
\bar{\psi}_{i} P \psi_{i+1} \quad\left(P=\gamma_{5}+r\right)
$$


is a $Q=1 / 3$ operator. At this layer, electric charge is not conserved. This is unacceptable and needs to be fixed.

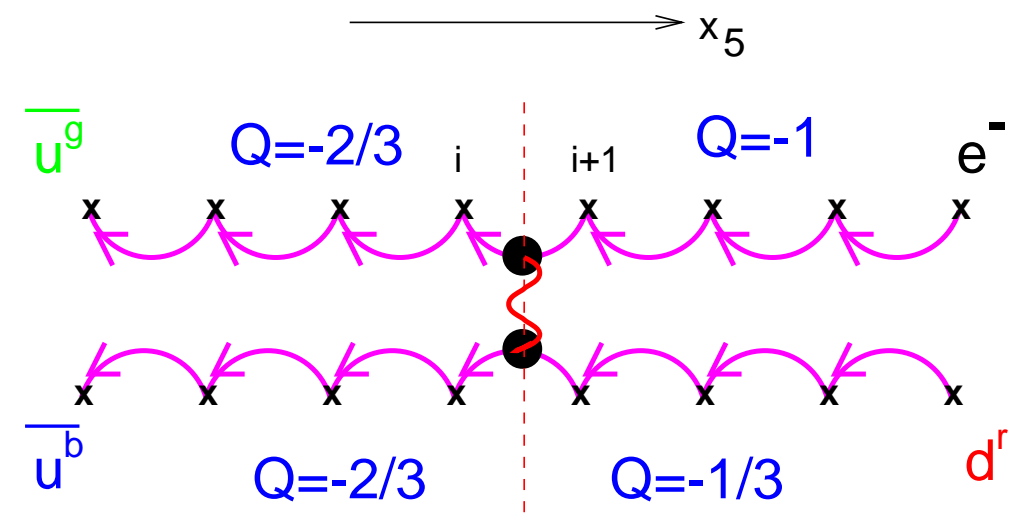

Fig. 26. Transferring charge between the doublets.

To restore the $U(1)$ symmetry one must transfer the charge from $\psi$ to the compensating doublet $\chi$. For this I replace the sum of hoppings with a product on the offending layer

$$
\bar{\psi}_{i} P \psi_{i+1}+\bar{\chi}_{i} P \chi_{i+1} \longrightarrow \bar{\psi}_{i} P \psi_{i+1} \times \bar{\chi}_{i} P \chi_{i+1} .
$$

This introduces an electrically neutral four Fermi operator. Note that it is baryon violating, involving a "lepto-quark/diquark" exchange, as sketched in Fig. (26). One might think of the operator as representing a "filter" at $x_{5}=i$ through which only charge compensating pairs of Fermions can pass. The need for such an operator in a fully finite theory was emphasized by Eichten and Preskill (1986), although the detailed scheme presented there had technical difficulties (Golterman, Petcher, and Rivas, 1993).

In five dimensions there is no chiral symmetry. Even for the free theory, combinations like $\bar{\psi}_{i} P \psi_{i+1}$ have vacuum expectation values. I use such as a "tadpole," with $\chi$ generating an effective hopping for $\psi$ and vice versa.

Actually the above four Fermion operator is not quite sufficient for all chiral anomalies, which can also involve right handed singlet Fermions. To correct this I need explicitly include the right handed sector, adding similar four Fermion couplings (also electrically neutral).

Having fixed the $U(1)$ of electromagnetism, I restore the strong $S U(3)$ with an antisymmetrization $Q^{r} Q^{g} Q^{b} \longrightarrow \epsilon^{\alpha \beta \gamma} Q^{\alpha} Q^{\beta} Q^{\gamma}$. Note that similar left-right inter-sector couplings are needed to correctly obtain the effects of topologically non-trivial strong gauge fields. 
An alternative view is to fold the lattice about the interior of the fifth dimension, placing all light modes on one wall and having the multi-Fermion operator on the other. This is the model of Creutz, Tytgat, Rebbi, and Xue (1997), with the additional intersector couplings correcting a technical error (Neuberger, 1997).

Unfortunately the scheme is still non rigorous. In particular, the non-trivial four Fermion coupling represents a new defect which should not give rise to unwanted extra zero modes. Note, however, that the five dimensional mass is the same on both sides of defect, removing topological reasons for such.

A second, and probably the biggest, concern is that the four Fermion coupling might induce an unwanted spontaneous symmetry breaking of one of the gauge symmetries. A paramagnetic phase without spontaneous symmetry breaking is needed. Creutz, Tytgat, Rebbi, and Xue (1997) showed that strongly coupled zero modes preserved the desired symmetries, but their analysis ignored contributions from heavy modes in the fifth dimension.

Assuming all works as desired, the model raises several other interesting questions. As formulated, I used a right handed neutrino to provide all quarks with partners. Is there some variation that avoids this particle, which completely decouples in the continuum limit? Another question concerns possible numerical simulations; is the effective action positive? Finally, I have used the details of the usual standard model, leaving open the question of whether this model is somehow special. Can one always use multi-Fermion couplings to eliminate undesired modes in other anomaly free chiral theories? 


\section{Chiral identities}

Returning to the domain-wall lattice formulation of the strong interactions, it may still be a bit mysterious how it is consistent for both pions and the $\eta^{\prime}$ to be made of the same surface modes and yet have rather different masses. Some intuition on this issue can be gained from some exact chiral identities. There are many equivalent ways to obtain similar relations; I pick one that appeals to me.

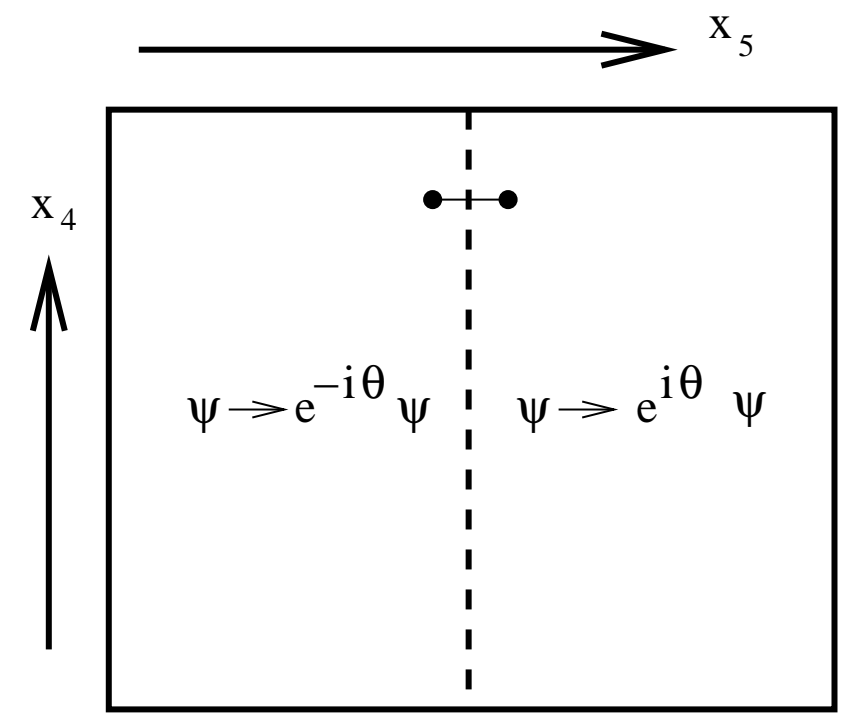

Fig. 27. Making opposite phase rotations on opposite sides of the box sets up an exact chiral symmetry relation. The bonds crossing the interface are not invariant under this change of variables.

To explore this, consider taking the simple domain-wall formulation on a five dimensional lattice and divide the sites in half with a hyper-plane in the middle of the fifth dimension. Put this hyper-plane between two sites in the fifth direction. Take all Fermionic variables on the "left" and multiply by a phase $e^{-i \theta / 2}$. Multiply all those fields on the "right" by $e^{i \theta / 2}$. This is sketched in Fig. (27) . Every term in the action is invariant except those hopping terms that cross the above hyper-plane. Since the measure is invariant under this transition, the path integral cannot change. This is equivalent to the statement that the exponential of the change in the action must have unit expectation value. Separating the exponential factors into terms even and odd in $\theta$, I have

$$
1=\left\langle\exp \left\{\sum_{x_{\mu}}\left(A\left(x_{\mu}\right)(\cos (\theta)-1)+B\left(x_{\mu}\right) \sin (\theta)\right)\right\}\right\rangle
$$


where $A$ and $B$ are operators only involving fields adjacent to the dividing hyper-plane and the sum is over the four dimensions of space-time. Explicitly, I define

$$
A=K_{5}\left(\bar{\psi}_{x_{5}+1}\left(1+\gamma_{5}\right) \psi_{x_{5}}+\bar{\psi}_{x_{5}}\left(1-\gamma_{5}\right) \psi_{x_{5}+1}\right)
$$

and

$$
B=i K_{5}\left(\bar{\psi}_{x_{5}+1}\left(1+\gamma_{5}\right) \psi_{x_{5}}-\bar{\psi}_{x_{5}}\left(1-\gamma_{5}\right) \psi_{x_{5}+1}\right)
$$

Here the hyperplane lies between $x_{5}$ and $x_{5}+1$, and $K_{5}$ is the hopping parameter in the extra dimension. Expanding to second order in $\theta$ and removing an overall spatial volume factor gives an exact result relating the expectation of $A$ with a vacuum susceptibility for the operator $B$

$$
\langle A(0)\rangle-\sum_{x_{\mu}}\left\langle B(0) B\left(x_{\mu}\right)\right\rangle=0 .
$$

If I average over positions, this is true gauge configuration by gauge configuration as well as at any volume.

Now consider two flavors and replace the phase factor by $e^{i \theta \tau_{3}}$ where $\tau_{3}$ is the Pauli matrix of isospin. Since $\tau_{3}^{2}=1$, the $\tau_{3}$ factor cancels out of the $A$ term. Corresponding to $B$, another operator appears

$$
B_{3}=i K_{5}\left(\bar{\psi}_{x_{5}+1}\left(1+\gamma_{5}\right) \tau_{3} \psi_{x_{5}}-\bar{\psi}_{x_{5}}\left(1-\gamma_{5}\right) \tau_{3} \psi_{x_{5}+1}\right)
$$

giving another exact relation

$$
\langle A(0)\rangle-\sum_{x_{\mu}}\left\langle B_{3}(0) B_{3}\left(x_{\mu}\right)\right\rangle=0 .
$$

Combining Eq. (82) with Eq. (84) shows that a singlet and a non-singlet susceptibility are exactly equal

$$
\left\langle B(0) B\left(x_{\mu}\right)\right\rangle=\left\langle B_{3}(0) B_{3}\left(x_{\mu}\right)\right\rangle .
$$

For a physical interpretation, consider the particles that can be created by this operator $B$. Because of isospin, $B_{3}$ has the quantum numbers to create a pion; call the coupling $g_{\pi}$. On the other hand, the operator $B$ can only create the singlet " $\eta^{\prime}$ " meson; call this coupling $g_{\eta^{\prime}}$. In the chiral limit, the former becomes light, giving a singularity in the right hand side of this equation. Inserting these intermediate states into Eq. (85) gives

$$
g_{\pi}^{2} / m_{\pi}^{2}=g_{\eta^{\prime}}^{2} / m_{\eta^{\prime}}^{2}+\ldots
$$


The "..." refers to heavier particles in the spectrum. As the chiral limit is approached, these operators deep in the fifth dimension have vastly different couplings to the physical particles on the surface

$$
g_{\eta^{\prime}}^{2} / g_{\pi}^{2} \sim m_{\eta^{\prime}}^{2} / m_{\pi}^{2} .
$$

This ratio can be qualitatively understood as a gluon exchange effect. The gluonic field doesn't know about the fifth dimension, so the operator $B$ can directly couple to it, which in turn can strongly couple to the quark modes on the walls. Since it is a flavor singlet, both quark flavors contribute equally to this coupling. On the other hand, for the flavor non-singlet case, the two flavors couple oppositely to the gauge field, and cancel out. In that case, the coupling to the surface modes must be through the Fermion fields, which have the exponential damping of the heavy mass in the fifth dimension.

This argument suggests that the coupling $g_{\eta^{\prime}}$ remains finite even in the infinite $L_{5}$ limit. In contrast, the expectation is for $g_{\pi}$ to go to zero with the pion mass, which should go to zero exponentially with $L_{5}$.

Note that the above exact relations can be kept exact when an explicit mass term is added. They just need corresponding additions to the operators $A$ and $B$. For the nonsinglet case these terms represent a form of the Gell-mann Oakes Renner relation (GellMann, Oakes, and Renner, 1968). The operators in the lattice center give exponentially suppressed corrections due to the discretization and make the relation exact.

With an explicit mass present it becomes possible in a hopping parameter expansion for a Fermion to travel first through the extra dimension and then directly back to the original wall via the mass term. An overall phase acquired in such a process gives the $\Theta$ parameter discussed in earlier sections. Whether the phase is directly in the mass itself or in the hoppings through the fifth dimension is a convention; the phase change implemented for the identity here moves this convention around. The first order transition expected at $\Theta=\pi$ shows explicitly that the phase acquired by a Fermion tunnelling through the extra dimension retains physical significance even in the infinite $L_{5}$ limit. 


\section{The Ginsparg-Wilson relation}

I now drop back to four dimensions and discuss a generic approach to chiral symmetry that has received considerable recent attention. This makes use of a class of lattice Dirac operators which generalize continuum chiral properties. Narayanan (1998) showed that this relation is closely related to the overlap approach, which in turn was motivated by the domain-wall scheme. This topic is rapidly evolving and is not the main thrust of this article. Nevertheless it is receiving sufficient attention that I will briefly discuss the central ideas, although this section will likely soon be out of date.

I begin by considering the Fermionic part of some action as a quadratic form

$$
S_{f}=\sum_{i} \bar{\psi} D \psi
$$

The usual "continuum" Dirac operator $D=\sum \gamma_{\mu} D_{\mu}$ naively anti-commutes with $\gamma_{5}$, i.e. $\left[\gamma_{5}, D\right]_{+}=0$. Then the change of variables $\psi \rightarrow e^{i \theta \gamma_{5}} \psi$ and $\bar{\psi} \rightarrow \bar{\psi} e^{i \theta \gamma_{5}}$ would be a symmetry of the action. This, however, is inconsistent with the chiral anomalies. The conventional continuum discussions map this phenomenon into the Fermionic measure (Fujikawa, 1979).

On the lattice we work with a finite number of degrees of freedom; thus, the above variable change is automatically a symmetry of the measure. To parallel the continuum discussion, it is necessary to modify the symmetry transformation so that the measure is no longer invariant. Remarkably, it is possible to construct actions exactly invariant under the altered symmetries.

To be specific, one particular modification (Neuberger, 1998b,c; Luscher, 1998; Chiu and Zenkin, 1999; Chandrasekharan, 1999) that leads to interesting consequences starts with the change of variables

$$
\begin{aligned}
& \psi \longrightarrow e^{i \theta \gamma_{5}(1+a D)} \psi \\
& \bar{\psi} \longrightarrow \bar{\psi} e^{i \theta \gamma_{5}}
\end{aligned}
$$

where $a$ represents the lattice spacing. Note the asymmetric way in which the independent Grassmann variables $\psi$ and $\bar{\psi}$ are treated. Inserting this into Eq. (88), and requiring the action to be unchanged gives the relation (Ginsparg and Wilson, 1982; Hasenfratz, Laliena, and Niedermayer, 1998; Hasenfratz, 1998)

$$
\gamma_{5} D+D \gamma_{5}+a D \gamma_{5} D=0
$$


I also assume the Hermeticity condition $\gamma_{5} D \gamma_{5}=D^{\dagger}$. The "Ginsparg-Wilson relation" in Eq. (90) along with the Hermititicity condition is equivalent to the unitarity of the combination $V=1+a D$.

Neuberger (1998b,c) and Chiu and Zenkin (1999) suggested a simple construction of an operator satisfying this condition. For this an appropriate operator $V$ could be found via a unitarization of an undoubled but chiral symmetry violating Dirac operator, such as the Wilson operator $D_{w}$ implicit in Eq. (10) . This operator should also satisfy the above Hermeticity condition. From this build

$$
V=-D_{w}\left(D_{w}^{\dagger} D_{w}\right)^{-1 / 2}
$$

More precisely, find a unitary operator to diagonalize $D_{w}^{\dagger} D_{w}$, take the square root of the eigenvalues, and undo this unitary transformation.

At this point the hopping parameter in $D_{w}$ is a parameter. To have the desired single light Fermion per flavor of the theory, the hopping parameter should be appropriately adjusted to lie above the critical value where $D_{w}$ describes a massless flavor, but not so large that additional doublers come into play (Neuberger, 1999; Golterman and Shamir, 2000). There are actually two parameters to play with, the hopping parameter of $D_{w}$, and the lattice spacing. When the latter is finite and gauge fields are present, the location of the critical hopping parameter in $D_{w}$ is expected to shift from that of the free Fermion theory. There is potentially a rather complex phase structure in the plane of these two parameters, with various numbers of doublers becoming exactly massless modes. The relation in Eq. (90) in and of itself does not in general determine the number of massless Fermions. In Section XVI I discussed a similar issue for domain-wall Fermions.

Although the Wilson operator entering this construction is local and quite sparse, the resulting action is not; it involves direct couplings between arbitrarily separated sites (Hernandez, Jansen and Luscher, 1999; Horvath, 1998, 1999). How rapidly these couplings fall with distance depends on the gauge fields and is not fully understood. The five dimensional domain-wall theory is local in the most naive sense of the word; all terms in the action only couple nearest neighbor sites. Were one to integrate out the heavy modes, however, the resulting low energy effective theory would also involve couplings with arbitrary range. Despite these non-localities, recent encouraging studies (Neuberger 1998c; Edwards, Heller, and Narayanan, 1999; Borici, 1999; Hernandez, Jansen and Lellouch, 2000; Dong, Lee, Liu, and Zhang, 2000; Gattringer, 2000) show that it may indeed be practical to implement the inversion implicit in Eq. (91) in large scale numerical simulations. The overlap operator 
should have memory advantages since a large number of fields corresponding to the extra dimension do not need to be stored. The extent to which this outweighs the additional complexity in implementation remains to be determined.

This approach hides the infinite sea of heavy Fermion states alluded to above. It is implicit in the presence of zero modes in the inversion in Eq. (91). This is directly related to the connection with the index theorems in the continuum; for recent reviews see Adams (2000) and Kerler (2000). Recent detailed analysis (Luscher, 2000; Kikukawa and Yamada, 1999) shows that this operator is particularly well behaved order by order in perturbation theory. This has led to hopes that this may lead the way to a rigorous formulation of chiral models, such as the standard model. 


\section{Speculations}

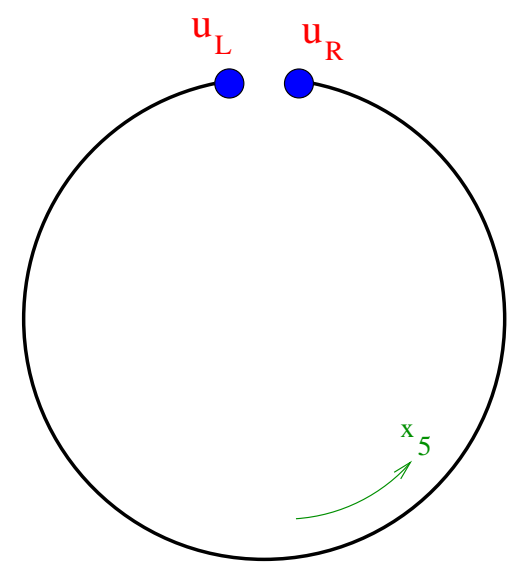

Fig. 28. Cutting a periodic fifth dimension gives the surfaces necessary for the topological zero modes.

I now ramble on with some general remarks about the basic domain-wall scheme. The existence of the end states relies on using open boundary conditions in the fifth direction. If I were to curl the extra dimension into a circle, they would be lost. To retrieve them, consider cutting such a circle, as in Fig. (28). Of course, if the size of the extra dimension is finite, the modes mix slightly. This is crucial for the scheme to accommodate anomalies, as discussed previously.

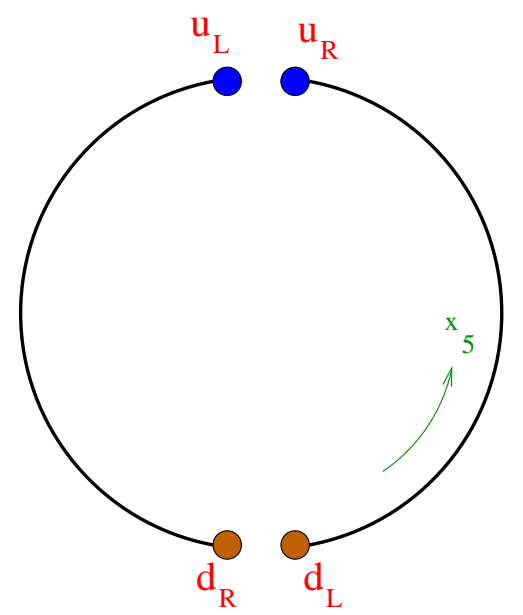

Fig. 29. Cutting the fifth dimension twice can give two flavors of quark.

Suppose I want a theory with two flavors of light Fermion, such as the up and down quarks. For this one might cut the circle twice, as shown in Fig. (29) . Remarkably, this construction keeps one chiral symmetry exact, even if the size of the fifth dimension is 
finite. Since the cutting divides the system into two completely disconnected pieces, in the notation of the figure, the number of $u_{L}+d_{R}$ particles is absolutely conserved. Similarly with $u_{R}+d_{L}$. Subtracting gives an exactly conserved axial charge corresponding to the continuum current

$$
j_{\mu 5}^{3}=\bar{\psi} \gamma_{\mu} \gamma_{5} \tau^{3} \psi
$$

The conservation holds even with finite $L_{5}$. There is a small flavor breaking since the $u_{L}$ mixes with the $d_{R}$. These symmetries are reminiscent of staggered (Kogut and Susskind, 1975) Fermions, where a single exact chiral symmetry is accompanied by a small flavor breaking. Now, however, the extra dimension gives additional control over the latter.

Despite this analogy, the situation is physically somewhat different in the zero applied mass limit. Staggered Fermions are expected to give rise to a single zero mass Goldstone pion, with the other pions acquiring mass through flavor breaking. In the doubly cut domain-wall picture, however, the zero mass limit has three degenerate equal mass particles as the lowest states. To see how this works it is simplest to discuss the physics in a chiral Lagrangian language. The finite fifth dimension generates an effective mass term, but it is not in a flavor singlet direction. Indeed, it is in a flavor direction orthogonal to the naive applied mass. In the usual "sombrero" picture of the effective Lagrangian, as illustrated in Fig. (6) and extensively discussed earlier, the two mass terms compete and the true vacuum rotates around the Mexican hat from the conventional "sigma" direction to the "pi" direction.

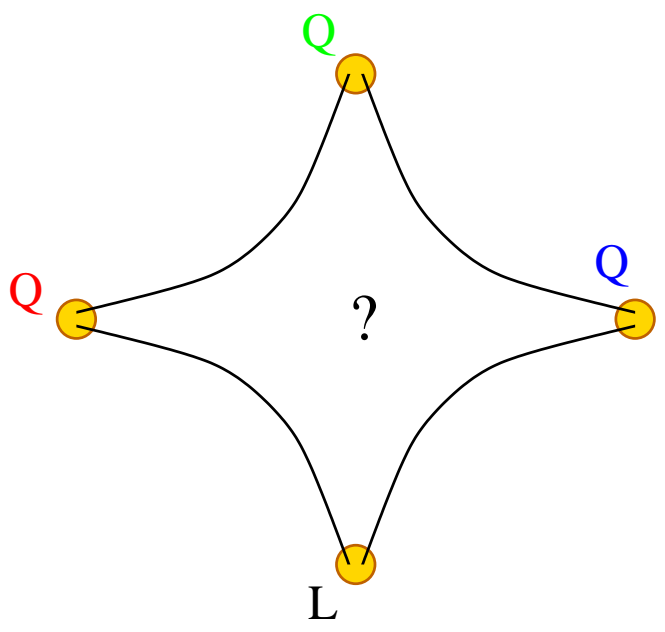

Fig. 30. Perhaps all species of Fermions are manifestations of a single field at various topological defects in a higher dimensional space.

Now I become more speculative. The idea of multiply cutting the fifth dimension to 
obtain several species suggests extensions to zero modes on more complicated manifolds. Multiple zero modes gives a mechanism to generate multiple flavors. Maybe one can have a theory where all the physical Fermions in four dimensions arise from a single Fermion field in the underlying higher dimensional theory. Schematically we might have something like Fig. (30) where each point represents some four dimensional surface and the question mark represents structures in the higher dimension that need specification. One nice feature provided by such a scheme is a possible mechanism for the transfer of various quantum numbers involved in anomalous processes. For example, the baryon non-conserving 't Hooft process ('t Hooft, 1976a,b) might arise from a lepton flavor tunneling into the higher manifold and reappearing on another surface as a baryon.

I've been rather abstract here. This generic mechanism is in fact the basis of the formulation of the standard model on the lattice (Creutz, Tytgat, Rebbi, and Xue, 1997) presented in Section XIX. For this model the question mark in the above figure is the four-Fermi interaction in the interior of the extra dimension. As emphasized by Neuberger (1997), that picture is closely tied with $S O(10)$ grand unified models. 


\section{Concluding remarks}

The last few years have seen remarkable progress towards the non-perturbative understanding of chiral symmetry in quantum field theory. This has largely been driven by the needs of lattice gauge theory, aided by techniques drawn from effective Lagrangians. The use of a tower of auxiliary Fermion fields provides a sink for the flow of Fermion states in anomalous processes. Formulation in terms of an extra dimension provides a useful analogy, and provides hints towards deeper connections with the higher dimensional theories in vogue today.

These approaches lead to greatly improved chiral behavior, such as the time honored relation $m_{Q} \sim m_{\pi}^{2}$. Encouraging exploratory calculations using both the domain-wall (Blum and Soni, 1997a,b; Chen et al., 2000; Blum et al., 2000). and the overlap (Neuberger 1998c; Edwards, Heller, and Narayanan, 1999; Borici, 1999; Hernandez, Jansen and Lellouch, 2000; Dong, Lee, Liu, and Zhang, 2000; Gattringer, 2000) formulations are underway. The new methods may soon dominate numerical lattice gauge simulations.

The progress has been particularly complete for vector-like theories, such as the strong interactions. Here the various methods, domain-wall/overlap/two cutoff, all appear to have essentially solved the confusion surrounding the early attempts to formulate chiral symmetry on the lattice. The situation is less clear when gauge fields are coupled to chiral currents, as in the standard model. Here formulations based overlap operator with the Ginsparg-Wilson relation appear promising; however, these schemes still remain somewhat formal. For the 't Hooft process it would be welcome to have a Hamiltonian discussion along the lines of section XVII to explicitly trace the transition of a baryon into a lepton in terms of a flow of states.

It remains unknown whether there exists a lattice formulation of the standard model which, in the cutoff form, maintains an exact local gauge symmetry and a finite number of degrees of freedom per unit volume. Models incorporating four Fermion couplings, such as presented in the section relating leptons and antiquarks, are an attempt to accomplish this. Unfortunately, such couplings are difficult to treat rigorously and these approaches remain controversial.

Chiral symmetry often plays a central role in extensions of the standard model. It has been argued (Narayanan and Neuberger, 1993a,b, 1994, 1995; Nishimura, 1987; Maru and Nishimura, 1998; Kaplan and Schmaltz, 2000) that the domain-wall approach also may provide a path to super-symmetry on the lattice. With adjoint Fermions in the domain- 
wall formalism, the states of one handedness can be removed via a Majorana mass term. This gives a theory where the low energy degrees of freedom are the gauge fields and a chiral set of adjoint fields. While the heavy modes severely violate super-symmetry, the low energy states represent exactly the spectrum of super-symmetric Yang-Mills theory, and do this without fine tuning. It would be interesting to see if these ideas can be extended to more general super-symmetric models.

This manuscript has been authored under contract number DE-AC02-98CH10886 with the U.S. Department of Energy. Accordingly, the U.S. Government retains a non-exclusive, royalty-free license to publish or reproduce the published form of this contribution, or allow others to do so, for U.S. Government purposes. The work was also supported by grants No. 95-00077 and No. 98-000302 from the United-states - Israel Binational Science foundation (BSF) Jerusalem, Israel, which enabled visits wherein many of the ideas discussed here were developed. 


\section{References}

Adams, D., 2000, e-Print Archive: hep-lat/0001014.

Adler, S. L., 1969, Phys. Rev. 117, 2426.

Alonso, J., Ph. Boucaud, J. Cortés, and E. Rivas, 1991, Phys. Rev. D44, 3258.

Ambjorn, J., J. Greensite, and C. Peterson, Nucl. Phys. B221 (1983) 381.

Aoki, S., 1989, Nucl. Phys. B314, 79.

Aoki, S., S. Boetcher, and A. Gocksch, 1994, Phys. Lett. B331, 157.

Aoki, S., and A. Gocksch, 1992, Phys. Rev. D45, 3845.

Aoki, S., A. Ukawa, and T. Umemura, 1996, Phys. Rev. Lett. 76, 873.

Banks, T., and A. Casher, 1980, Nucl. Phys. B169, 103.

Bell, J.S. and R. Jackiw, 1969, Nuovo Cimento 60A, 47.

Bijnens, J., J. Prades, and E. de Rafael, 1995, Phys.Lett. B348 226-238.

Bitar, K., and P. Vranas, 1994a, Phys. Rev. D50, 3406.

Bitar, K., and P. Vranas, 1994b, Nucl. Phys. B, Proc. Suppl. 34, 661 (1994).

Blum, T., P. Chen, N. Christ, C. Cristian, C. Dawson, G. Fleming, A. Kaehler, X. Liao, G. Liu, C. Malureanu, R. Mawhinney, S. Ohta, G. Siegert, A. Soni, C. Sui, P. Vranas, M. Wingate, L. Wu and Y. Zhestkov, 2000, e-Print Archive: hep-lat/0007038.

Blum, T., and A. Soni, 1997a, Phys. Rev. Lett. 79, 3595.

Blum, T., and A. Soni, 1997b, Phys. Rev. D56, 174.

Bock, W., M. Golterman, and Y. Shamir, 1998a, Phys. Rev. Lett. 80, 3444.

Bock, W., M. Golterman, and Y. Shamir, 1998b, Phys. Rev. D58, 034501 (1998).

Bodwin,G., 1996, Phys.Rev.D54:6497-6520.

Borici A., 1999, e-Print Archive: hep-lat/9910045.

Borrelli, A., L. Maiani, R. Sisto, G.C. Rossi, and M. Testa, 1990, Nucl.Phys. B333 335.

Brower, R., and B. Svetitsky, 2000, Phys. Rev. D61, 114511.

Callan, C., and J. Harvey, 1985, Nucl. Phys. B250, 427.

Chandrasekharan, S., 1999, Phys. Rev. D60, 074503.

Chen, P. N. Christ, G. Fleming, A. Kaehler, C. Malureanu, R. Mawhinney, G. Siegert, C. Sui, L. Wu, Y. Zhestkov, and P. Vranas, 2000, e-Print Archive: hep-lat/0006010.

Chiu, T. and S. Zenkin, 1999, Phys. Rev. D59, 074501.

Coleman, S., 1976, Annals Phys. 101, 239.

Coleman, S., and B. Grossman, 1982, Nucl. Phys. B203, 205.

Coleman, S. and E. Weinberg, 1973, Phys. Rev. D7, 1888.

Creutz, M., 1983, Quarks Gluons and Lattices (Cambridge). 
Creutz, M., 1995a, Phys. Rev. D52, 2951 (1995).

Creutz, M., 1995b, Nucl. Phys. B (Proc. Suppl.) 42, 56.

Creutz, M., 1997, e-Print Archive: hep-lat/9608024, in RHIC Summer Study '96: Theory workshop on relativistic heavy ion collisions, D. Kahana and Y. Pang, eds., pp. 49-54 (NTIS).

Creutz, M., 1999, Phys. Rev. Letters 83, 2636.

Creutz, M. and I. Horvath, 1994, Phys. Rev. D50, 2297.

Creutz, M., and M. Tytgat, 1996, Phys. Rev. Letters 76, 4671.

Creutz, M., M. Tytgat, C. Rebbi, S.-S. Xue, 1997, Phys. Lett. B402, 341.

Dashen, R., 1971, Phys. Rev. D3, 1879 (1971).

Distler, J., and S. Rey, 1993, e-Print Archive: hep-lat/9305026.

Dong, S., F. Lee, K. Liu, and J. Zhang, 2000, e-Print Archive: hep-lat/0006004.

Donoghue, J., B. Holstein, and D. Wyler, 1992, Phys. Rev. Lett. 69, 3444.

Drell, S., M. Weinstein, and S. Yankielowicz, 1976, Phys. Rev. D19, 3698.

Edwards, R., U. Heller, R. Narayanan, 1999, Phys. Rev. D59, 094510;

Eichten, E. and J. Preskill, 1986, Nucl. Phys. B268, 179.

Evans, N., S. Hsu, A. Nyffeler, and M. Schwetz, 1997, Nucl. Phys. B494, 200.

Friedberg, R., T.D. Lee, and Y. Pang, 1994, J. Math. Phys. 35, 5600.

Frolov, S. and A. Slavnov, 1994, Nucl. Phys. B411, 647.

Fujikawa, K., 1979 Phys. Rev. Lett. 42, 1195.

Furman, V., and Y. Shamir, 1995, Nucl. Phys. B439, 54.

Gattringer, C., 2000, e-Print Archive: hep-lat/0003005.

Gell-Mann, M., R. Oakes, and B. Renner, 1968, Phys. Rev. 175, 2195 (1968).

Ginsparg, P. and K. Wilson, 1982, Phys. Rev. D25, 2649.

Gockeler, M., A. Kronfeld, G. Schierholz, and U.J. Wiese, 1993, Nucl. Phys. B404, 839.

Goldstone, J., and F. Wilczek, 1981, Phys. Rev. Lett. 47, 1986.

Golterman, M., K. Jansen, D. Petcher, and J. Vink, 1994, Phys. Rev. D49, 1606.

Golterman, M., D. Petcher, and E. Rivas, 1993, Nucl. Phys. B395, 596.

Golterman, M., and Y. Shamir, 1995, Phys. Rev. D51, 3026.

Golterman, M., and Y. Shamir, 1999, e-Print Archive: hep-lat/0007021.

Hasenfratz, P., 1998, Nucl. Phys. B525, 401.

Hasenfratz, P., V. Laliena, and Ferenc Niedermayer, 1998, Phys. Lett. B427, 125.

Hernandez, P., and P. Boucaud, 1998, Nucl. Phys. B513, 593.

Hernandez, P., K. Jansen, and Martin Luscher, 1999, Nucl. Phys. B552, 363. 
Hernandez, P, K. Jansen, and L. Lellouch, 2000, e-Print Archive: hep-lat/0001008;

Hernandez, P. and R. Sundrum, 1995, Nucl. Phys. B455, 287.

Holstein, B., 1993, Am. J. Phys. 61, 142.

't Hooft, G., 1976a, Phys. Rev. Lett. 37, 8 (1976).

't Hooft, G., 1976b, Phys. Rev. D14, 3432 (1976).

't Hooft, G., 1986, Phys. Rept. 142, 357.

't Hooft, G., 1995, Phys. Lett. B349, 491.

Horvath, I, 1998, Phys. Rev. Lett. 81, 4063 (1998);

Horvath, I, 1999, Phys. Rev. D60, 034510 (1999).

Hsu, S., 1995, preprint YCTP-P5-95.

Jackiw,R. and C. Rebbi, 1976, Phys. Rev. D13, 3398.

Jansen, K., and M. Schmaltz, 1992, Phys. Lett. B296, 374.

Kaplan, D., 1992, Phys. Lett. B288, 342.

Kaplan, D., and M. Schmaltz, 2000, e-Print Archive: hep-lat/0002030.

Karsten, L., and J. Smit, 1981, Nucl. Phys. B183, 103.

Kelvin, Lord, 1904, Baltimore lectures on molecular dynamics and the wave theory of light (Clay, London).*

Kerler, W., 2000, e-Print Archive: hep-lat/0007023.

Kikukawa, Y., 1999, e-Print Archive: hep-lat/9912056.

Kikukawa, Y., and A. Yamada, 1999, Phys. Lett. B448, 265.

Kogut, J. and L. Susskind, 1975, Phys. Rev. D11, 395.

Kronfeld, A., 1995, e-Print Archive: hep-lat/9504007;

Lee, I-H., and R. Schrock, 1988, Nucl. Phys. B305, 305.

Leutwyler, H., 1990, Nucl. Phys. B337 108.

Luscher, M, 1998, Phys.Lett.B428, 342.

Luscher, M., 2000, JHEP 0006, 028.

Maru, N., and J. Nishimura, 1998, Int. J. Mod. Phys. A13, 2841.

Montvay, I., 1987, Phys. Lett. 199B, 89.

Montvay, I., 1993, Nucl. Phys. B (Proc. Suppl.) 30, 621.

* Kelvin extensively edited these lectures from the original version reprinted in Kelvin's Baltimore lectures and modern theoretical physics (MIT, 1987). In the latter the word "chiral" is not mentioned, but Thomson was clearly struggling for a term when on page 186 he writes "I have objected to the name rotary, because it is not properly applied, and have taken the name helical because the phenomenon essentially depends on a screw like form somehow or other." 
Narayanan, R., 1998, Phys. Rev. D58, 097501.

Narayanan, R. and H. Neuberger, 1993a, Phys. Lett. B302, 62.

Narayanan, R. and H. Neuberger, 1993b, Phys. Rev. Lett. 71, 3251;

Narayanan, R. and H. Neuberger, 1994, Nucl. Phys. B412, 574.

Narayanan, R. and H. Neuberger, 1995, Nucl. Phys. B443, 305.

Neuberger, H., 1997, Phys. Lett. B413, 387.

Neuberger, H, 1998a, Phys. Rev. Lett. 81, 4060;

Neuberger, H., 1998b Phys. Lett. B417, 141.

Neuberger, H., 1998c, Phys. Lett. B427 353.

Neuberger, H., 1999, e-Print Archive: hep-lat/9911022.

Nielsen, H., and M. Ninomiya, 1981a, Phys. Lett. B105, 219.

Nielsen, H., and M. Ninomiya, 1981b, Nucl. Phys. B185, 20, (Erratum: 1982 ibid. B195, $541)$.

Nielsen, H., and M. Ninomiya, 1981c, Nucl. Phys. B193, 173.

Nishimura, J., 1997, Phys. Lett. B406, 215.

Raffelt, G., 1990, Phys. Rept. 198, 1.

Randjbar-Daemi, S., and J. Strathdee, 1996a, Nucl. Phys. B461, 305.

Randjbar-Daemi, S., and J. Strathdee, 1996b, Nucl. Phys. B466, 335.

Seiler, E., and I. Stamatescu, 1982, Phys. Rev. D25, 2177.

Shamir, Y., 1993a, Phys. Rev. Lett. 71, 2691.

Shamir, Y., 1993b, Nucl. Phys. B406, 90.

Shamir, Y., 1994, Nucl. Phys. B417, 167 (1994).

Sharpe, S., and R. Singleton, 1998, Phys. Rev. D58, 074501.

Shockley, W., 1939, Phys. Rev. 56317.

Smilga, A., 1999, Phys. Rev. D59, 114021.

Smit, J., 1980, Nucl. Phys. B175 307 (1980).

Svetitsky, B., S. Drell, H. Quinn, and M. Weinstein, 1980, Phys. Rev. D22, 490.

Turner, M., 1990, Phys. Rept. 197, 67.

Tytgat, M., 1999, e-Print Archive: hep-ph/9909532.

van Baal, P., 1998, Nucl. Phys. Proc. Suppl. 63, 126.

Verbaarschot,J., 1994, Phys. Rev. Lett. 72, 2531.

Wess, J., and B. Zumino, 1971, Phys. Lett. 37B, 95.

Wilson, K., 1977, in New Phenomena in Subnuclear Physics, edited by A. Zichichi (Plenum Press, N. Y.). 
Witten, E., 1983a, Nucl. Phys. B223, 422.

Witten, E., 1983b, Nucl. Phys. B223, 433.

Witten, E., 1984, Commun. Math. Phys. 92, 455. 